\title{
Itô's Formula, the Stochastic Exponential, and Change of Measure on General Time Scales
}

\author{
Wenqing Hu \\ Department of Mathematics and Statistics, Missouri University of Science and Technology (Formerly University of Missouri, Rolla), \\ Rolla, MO, USA \\ Correspondence should be addressed to Wenqing Hu; huwen@mst.edu
}

Received 8 December 2016; Revised 28 March 2017; Accepted 29 March 2017; Published 18 April 2017

Academic Editor: Allan Peterson

Copyright (C) 2017 Wenqing Hu. This is an open access article distributed under the Creative Commons Attribution License, which permits unrestricted use, distribution, and reproduction in any medium, provided the original work is properly cited.

We provide an Itô formula for stochastic dynamical equation on general time scales. Based on this Itô's formula we give a closedform expression for stochastic exponential on general time scales. We then demonstrate Girsanov's change of measure formula in the case of general time scales. Our result is being applied to a Brownian motion on the quantum time scale ( $q$-time scale).

\section{Introduction}

The theory of dynamical equation on time scales ([1]) has attracted many researches recently. In particular, attempts of extension to stochastic dynamical equations and stochastic analysis on general time scales have been made in several previous works $([2-6])$. In the work [3] the authors mainly work with a discrete time scale; in [2] the authors introduce an extension of a function and define the stochastic as well as deterministic integrals as the usual integrals for the extended function; in [4] the authors make use of their results on the quadratic variation of a Brownian motion ([7]) on time scales and, based on this, they define the stochastic integral via a generalized version of the Itô isometry; in [6] the authors introduce the so-called $\nabla$-stochastic integral via the backward jump operator and they also derive an Itô formula based on this definition of the stochastic integral. We notice that different previous works adopt different notions of the stochastic integral and there lacks a uniform and coherent theory of a stochastic calculus on general time scales.

The purpose of the present article is to fill in this gap. We will be mainly working under the framework of [2], in that we define our stochastic integral using the definition given in [2]. We then present a general Itô's formula for stochastic dynamical equations under the framework of [2]. Our Itô formula works for general time scales and thus fills the gap left in [3], which deals with only discrete time scales. By making use of Itô's formula we obtain a closed-form expression for the stochastic exponential on general time scales. We will then demonstrate a change of measure (Girsanov's) theorem for stochastic dynamical equation on time scales.

We would like to point out that our change of measure formula is different from the continuous process case in that the density function is not given by the stochastic exponential but rather is found by the fact that the process on the time scale can be extended to a continuous process simply by linear extension.

It is also worth mentioning that our construction is different from [8] in that we are working with the case that the time parameter of the process is running on a time scale, whereas in [8] and related works (e.g., [9-11]) the authors are working with the case that the state space of the process is a time scale.

We note that stochastic calculus on the so-called $q^{-}$ Brownian motion has been considered in [12-14]. As an application, we will also work our Itô formula for a Brownian motion on the quantum time scale ( $q$-time scale) case at the last section of the paper.

The paper is organized as follows. In Section 2 we discuss some basic set-up for time scales calculus. In Section 3 we will briefly review the results in [2] and define the stochastic integral and stochastic dynamical equation on time scales. In Section 4 we present and prove our Itô formula. In Section 5 we discuss the formula for stochastic exponential. In Section 6 we prove the change of measure (Girsanov's) formula. Finally in Section 7 we consider an example of Brownian motion on a quantum time scale. 


\section{Set-Up: Basics of Time Scales Calculus}

A time scale $\mathbb{T}$ is an arbitrary nonempty closed subset of the real numbers $\mathbb{R}$, where we assume that $\mathbb{T}$ has the topology that it inherits from the real numbers $\mathbb{R}$ with the standard topology.

We define the forward jump operator by

$$
\sigma(t)=\inf \{s \in \mathbb{T}: s>t\}
$$

$\forall t \in \mathbb{T}$ such that this set is nonempty,

and the backward jump operator by

$$
\begin{aligned}
& \rho(t)=\sup \{s \in \mathbb{T}: s<t\} \\
& \forall t \in \mathbb{T} \text { such that this set is nonempty. }
\end{aligned}
$$

Let $t \in \mathbb{T}$. If $\sigma(t)>t$, then $t$ is called right-scattered. If $\sigma(t)=t$, then $t$ is called right-dense. If $\rho(t)<t$, then $t$ is called left-scattered. If $\rho(t)=t$, then $t$ is called left-dense. Moreover, the sets $\mathbb{T}^{\kappa}$ and $\mathbb{T}_{\kappa}$ are derived from $\mathbb{T}$ as follows: if $\mathbb{T}$ has a left-scattered maximum, then $\mathbb{T}^{\kappa}$ is the set $\mathbb{T}$ without that left-scattered maximum; otherwise, $\mathbb{T}^{\mathcal{K}}=\mathbb{T}$. If $\mathbb{T}$ has a right-scattered minimum, then $\mathbb{T}_{\kappa}$ is the set $\mathbb{\mathbb { V }}$ without that right-scattered minimum; otherwise, $\mathbb{T}_{\kappa}=\mathbb{T}$. The graininess function is defined by $\mu(t)=\sigma(t)-t$ for all $t \in \mathbb{T}^{\kappa}$.

Notice that since $\mathbb{T}$ is closed, for any $t \in \mathbb{T}$, the points $\sigma(t)$ and $\rho(t)$ are belonging to $\mathbb{T}$.

For a set $A \subset \mathbb{R}$ we denote the set $A_{\mathbb{T}}=A \cap \mathbb{T}$.

Given a time scale $\mathbb{T}$ and a function $f: \mathbb{T} \rightarrow \mathbb{R}$, the delta (or Hilger) derivative $f^{\Delta}(t)$ of $f$ at $t \in \mathbb{T}$ is defined as follows ([1, Definition 1.10]).

Definition 1. Assume $f: \mathbb{T} \rightarrow \mathbb{R}$ is a function and let $t \in$ $\mathbb{T}^{\mathcal{K}}$. Then we define $f^{\Delta}(t)$ to be the number (provided that it exists) with the property that, given any $\varepsilon>0$, there is a neighborhood $U$ of $t$ (i.e., $U=(t-\delta, t+\delta) \cap \mathbb{T}$ for some $\delta>0$ ) such that

$$
\begin{array}{r}
\left|[f(\sigma(t))-f(s)]-f^{\Delta}(t)[\sigma(t)-s]\right| \leq \varepsilon|\sigma(t)-s| \\
\forall s \in U .
\end{array}
$$

The delta derivative is characterized by the following theorem [1, Theorem 1.16].

Theorem 2. Assume that $f: \mathbb{T} \rightarrow \mathbb{R}$ is a function and let $t \in \mathbb{T}^{\kappa}$. Then one has the following:

(i) if $f$ is differentiable at $t$, then $f$ is continuous at $t$.

(ii) if $f$ is continuous at $t$ and $t$ is right-scattered, then $f$ is differentiable at $t$ with

$$
f^{\Delta}(t)=\frac{f(\sigma(t))-f(t)}{\sigma(t)-t} .
$$

(iii) Ift is right-dense, then $f$ is differentiable at $t$ if and only if the limit

$$
\lim _{s \rightarrow t} \frac{f(t)-f(s)}{t-s}
$$

exists as a finite number. In this case

$$
f^{\Delta}(t)=\lim _{s \rightarrow t} \frac{f(t)-f(s)}{t-s} .
$$

(iv) If $f$ is differentiable at $t$, then

$$
f(\sigma(t))=f(t)+\mu(t) f^{\Delta}(t) .
$$

\section{Stochastic Integrals and Stochastic Differential Equations on Time Scales}

We will adopt the definitions introduced in [2] as our definition of a Brownian motion and Itô's stochastic integral on time scales. In the next section we will derive an Itô formula corresponding to the stochastic integral defined in such a way.

Definition 3. A Brownian motion indexed by a time scale $\mathbb{T} \subset \mathbb{R}$ is an adapted stochastic process $\left\{W_{t}\right\}_{t \in \mathbb{T} \cup\{0\}}$ on a filtered probability space $\left(\Omega, \mathscr{F}_{t}, \mathbf{P}\right)$ such that

(1) $\mathbf{P}\left(W_{0}=0\right)=1$;

(2) if $s<t$ and $s, t \in \mathbb{T}$, then the increment $W_{t}-W_{s}$ is independent of $\mathscr{F}_{s}$ and is normally distributed with mean 0 and variance $t-s$;

(3) the process $W_{t}$ is almost surely continuous on $\mathbb{T}$.

Note that property (3) is proved in the work [5].

For a random function $f:[0, \infty)_{\mathbb{T}} \times \Omega \rightarrow \mathbb{R}$ we define the extension $\tilde{f}:[0, \infty) \times \Omega \rightarrow \mathbb{R}$ by

$$
\tilde{f}(t, \omega)=f\left(\sup [0, t]_{\mathbb{T}}, \omega\right)
$$

for all $t \in[0, \infty)$.

We shall make use of the definitions given in [2] for the classical Lebesgue and Riemann integral. For any random function $f:[0, \infty)_{\mathbb{T}} \times \Omega \rightarrow \mathbb{R}$ and $T<\infty$ we define its $\Delta$ Riemann (Lebesgue) integral as

$$
\int_{0}^{T} f(t, \omega) \Delta t=\int_{0}^{T} \tilde{f}(t, \omega) d t,
$$

where the integral on the right-hand side of the above equation is interpreted as a standard Riemann (Lebesgue) integral. In a similar way, the work [2] defines a stochastic integral for an $L^{2}\left([0, T]_{\mathbb{T}}\right)$-progressively measurable random function $f(t, \omega)$ as

$$
\int_{0}^{T} f(t, \omega) \Delta W_{t}=\int_{0}^{T} \tilde{f}(t, \omega) d W_{t},
$$

where again the right-hand side of the above equation is interpreted as a standard Itô stochastic integral. Note that the way (8) in which we define the extension guarantees that the function $\tilde{f}(t, \omega)$ is progressively measurable.

In [2] the authors then defined the solution of the $\Delta$ stochastic differential equation indicated by the notation

$$
\Delta X_{t}=b(t, X) \Delta t+\sigma(t, X) \Delta W_{t} \text {, }
$$


as the process $\left\{X_{t}\right\}_{t \in[0, T]_{\pi}}$ such that

$$
X_{t_{2}}-X_{t_{1}}=\int_{t_{1}}^{t_{2}} b\left(t, X_{t}\right) \Delta t+\int_{t_{1}}^{t_{2}} \sigma\left(t, X_{t}\right) \Delta W_{t}
$$

with the deterministic and stochastic integrals on the righthand side of the above equality interpreted as was just mentioned. Under the condition of continuity in the $t$ variable and uniform Lipschitz continuity in the $x$-variable of the functions $b(t, x)$ and $\sigma(t, x)$, together with being no worse than linear growth in $x$-variable, existence and pathwise uniqueness of strong solution to (11) are proved in [2].

\section{Itô's Formula for Stochastic Integrals on Time Scales}

We will make use of the following fact that is simple to prove.

Proposition 4. The set of all left-scattered or right-scattered

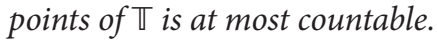

Proof. If $x \in \mathbb{T}$ is a right-scattered point, then $I_{x}=(x, \sigma(x))$ is an open interval such that $I_{x} \cap \mathbb{\mathbb { T }}=\emptyset$. Similarly, if $x \in \mathbb{T}$ is a left-scattered point, then $I_{x}=(\rho(x), x)$ is an open interval such that $I_{x} \cap \mathbb{T}=\emptyset$. Suppose $x<y$ and $x, y \in \mathbb{T}$. We then distinguish four different cases.

Case 1 (both $x$ and $y$ are right-scattered). We argue that in this case we have $I_{x} \cap I_{y}=\emptyset$. Suppose this is not the case, then we must have $\sigma(x)>y$. But we see that $\sigma(x)=\inf \{s>$ $x: s \in \mathbb{T}\}$ and $y \in \mathbb{T}$. So we must have $\sigma(x) \leq y$. We arrive at a contradiction.

Case 2 (both $x$ and $y$ are left-scattered). This case is similar to Case 1 and we conclude that $I_{x} \cap I_{y}=\emptyset$.

Case 3 ( $x$ is left-scattered; $y$ is right-scattered). In this case we see that $I_{x}=(\rho(x), x)$ and $I_{y}=(y, \sigma(y))$, as well as $x<y$. This implies that $I_{x} \cap I_{y}=\emptyset$.

Case 4 ( $x$ is right-scattered; $y$ is left-scattered). In this case $I_{x}=(x, \sigma(x))$ and $I_{y}=(\rho(y), y)$. If $\sigma(x) \leq \rho(y)$, then $I_{x} \cap$ $I_{y}=\emptyset$. If $\sigma(x)>\rho(y)$, then we see that $(x, y)=I_{x} \cup I_{y}$ so that $(x, y) \cap \mathbb{\mathbb { V }}=\emptyset$. That implies further that $\sigma(x)=y$ and $\rho(y)=x$; that is, $I_{x}=I_{y}$.

Thus we see that for all points $x \in \mathbb{T}$ being left- or right-scattered, the set of all open intervals of the form $I_{x}$ are disjoint subsets of $\mathbb{R}$. Henceforth there are at most countably many such intervals. Each such interval corresponds to one or two endpoints in $\mathbb{T}$ that are either left- or right-scattered. Thus the total number of left- or right-scattered points in $\mathbb{T}$ is at most countably many.

Let $C$ be the (at most) countable set of all left-scattered or right-scattered points of $\mathbb{T}$. As we have already seen in the proof of the previous proposition, the set $C$ corresponds to at most countably many open intervals $\mathscr{I}=\left\{I_{1}, I_{2}, \ldots\right\}$ such that (1) for any $k \neq l, I_{k} \cap I_{l}=\emptyset$; (2) either the left-endpoint or right-endpoint or both endpoints of any of the $I_{k}$ 's are in
$\mathbb{T}$ and are left- or right-scattered; (3) $I_{k} \cap \mathbb{T}=\emptyset$ for any $k=1,2, \ldots ;(4)$ any point in $C$ is a left- or right-endpoint of one of the $I_{k}$ 's.

We will denote $I_{k}=\left(s_{I_{k}}^{-}, s_{I_{k}}^{+}\right)$. Since, for any $x \in \mathbb{T}$, the points $\sigma(x)$ and $\rho(x)$ are in $\mathbb{T}$, we further infer that, for any such interval $I_{k}$, we have the fact that $s_{I_{k}}^{-}$and $s_{I_{k}}^{+}$are in $\mathbb{T}$, so that $s_{I_{k}}^{-}$is right-scattered and $s_{I_{k}}^{+}$is left-scattered.

We then establish the following Itô formula.

For any two points $t_{1}, t_{2} \in \mathbb{T}, t_{1} \leq t_{2}$, and any open interval $I_{k} \in \mathscr{I}$, such that $I_{k} \cap\left[t_{1}, t_{2}\right] \neq \emptyset$, we have $I_{k} \subset\left(t_{1}, t_{2}\right)$. This is because if that is not the case, then $t_{1}$ or $t_{2}$ will belong to $I_{k}$, contradictory to the fact that $I_{k} \cap \mathbb{T}=\emptyset$. We conclude that

$$
\left\{I_{k} \in \mathscr{I}: I_{k} \cap\left[t_{1}, t_{2}\right] \neq \emptyset\right\}=\left\{I_{k} \in \mathscr{I}: I_{k} \subset\left(t_{1}, t_{2}\right)\right\} .
$$

Let us consider a function $f(t, x): \mathbb{T} \times \mathbb{R} \rightarrow \mathbb{R}$. Let $f^{\Delta}(t, x), f^{\Delta^{2}}(t, x)$ be the first- and second-order delta (Hilger) derivatives of $f$ with respect to time variable $t$ at $(t, x)$ and let $(\partial f / \partial x)(t, x)$ and $\left(\partial^{2} f / \partial x^{2}\right)(t, x)$ be the first- and second-order partial derivatives of $f$ with respect to space variable $x$ at $(t, x)$.

Theorem 5 (Itô's formula). Let any function $f: \mathbb{T} \times \mathbb{R} \rightarrow \mathbb{R}$ be such that $f^{\Delta}(t, x), f^{\Delta^{2}}(t, x),(\partial f / \partial x)(t, x),\left(\partial^{2} f / \partial x^{2}\right)(t, x)$, $\left(\partial f^{\Delta} / \partial x\right)(t, x)$, and $\left(\partial^{2} f^{\Delta} / \partial x^{2}\right)(t, x)$ are continuous on $\mathbb{T} \times \mathbb{R}$. Set any $t_{1} \leq t_{2}, t_{1}, t_{2} \in[0, \infty)_{\mathbb{T}}$; then we have

$$
\begin{aligned}
& f\left(t_{2}, W_{t_{2}}\right)-f\left(t_{1}, W_{t_{1}}\right)=\int_{t_{1}}^{t_{2}} f^{\Delta}\left(s, W_{s}\right) \Delta s \\
& +\int_{t_{1}}^{t_{2}} \frac{\partial f}{\partial x}\left(s, W_{s}\right) \Delta W_{s}+\frac{1}{2} \int_{t_{1}}^{t_{2}} \frac{\partial^{2} f}{\partial x^{2}}\left(s, W_{s}\right) \Delta s \\
& +\sum_{I_{k} \in \mathcal{F}, I_{k} c\left(t_{1}, t_{2}\right)}\left[f\left(s_{I_{k}}^{+}, W_{s_{I_{k}}^{+}}\right)-f\left(s_{I_{k}}^{+}, W_{s_{I_{k}}^{-}}\right)\right. \\
& -\frac{\partial f}{\partial x}\left(s_{I_{k}}^{-}, W_{s_{I_{k}}^{-}}\right)\left(W_{s_{I_{k}}^{+}}-W_{s_{I_{k}}^{-}}\right) \\
& \left.-\frac{1}{2} \frac{\partial^{2} f}{\partial x^{2}}\left(s_{I_{k}}^{-}, W_{s_{I_{k}}^{-}}\right)\left(s_{I_{k}}^{+}-s_{I_{k}}^{-}\right)\right] .
\end{aligned}
$$

Proof. We will make use of the following classical version (Peano form) of Taylor's theorem: for any function $f: \mathbb{T} \times$ $\mathbb{R} \rightarrow \mathbb{R}$ such that $(\partial f / \partial x)(t, x)$ and $\left(\partial^{2} f / \partial x^{2}\right)(t, x)$ are continuous on $\mathbb{T} \times \mathbb{R}$, and any $s \in \mathbb{T}$ and $x_{1}, x_{2} \in \mathbb{R}$, we have

$$
\begin{aligned}
f\left(s, x_{2}\right)-f\left(s, x_{1}\right)= & \frac{\partial f}{\partial x}\left(s, x_{1}\right)\left(x_{2}-x_{1}\right) \\
& +\frac{1}{2} \frac{\partial^{2} f}{\partial x^{2}}\left(s, x_{1}\right)\left(x_{2}-x_{1}\right)^{2} \\
& +R_{C}^{f}\left(s ; x_{1}, x_{2}\right)
\end{aligned}
$$


where

$$
\left|R_{C}^{f}\left(s ; x_{1}, x_{2}\right)\right| \leq r\left(\left|x_{2}-x_{1}\right|\right)\left(x_{2}-x_{1}\right)^{2}
$$

and $r: \mathbb{R}_{+} \rightarrow \mathbb{R}_{+}$is an increasing function with $\lim _{u \downarrow 0} r(u)=$ 0 .

We will also make use of the time scale Taylor formula (see [1, Theorem 1.113] as well as [15]) applied to $f(t, x)$ up to first order in $t$ : for any $s_{2}>s_{1}$ and $s_{1}, s_{2} \in \mathbb{T}$; we have

$$
\begin{aligned}
f\left(s_{2}, x\right)-f\left(s_{1}, x\right)= & f^{\Delta}\left(s_{1}, x\right)\left(s_{2}-s_{1}\right) \\
& +R_{\mathrm{TS}}^{f}\left(x ; s_{1}, s_{2}\right),
\end{aligned}
$$

where

$$
\begin{aligned}
\left|R_{\mathrm{TS}}^{f}\left(x ; s_{1}, s_{2}\right)\right| & =\left|\int_{s_{1}}^{\rho\left(s_{2}\right)}\left(s_{2}-\sigma(s)\right) f^{\Delta^{2}}(s) \Delta s\right| \\
& \leq r\left(\left|s_{2}-s_{1}\right|\right)\left|s_{2}-s_{1}\right|
\end{aligned}
$$

with $r(\bullet)$ as before.

Combining (15) and (17) we see that we have

$$
\begin{aligned}
& f\left(s_{2}, x_{2}\right)-f\left(s_{1}, x_{1}\right)=\left[f\left(s_{2}, x_{2}\right)-f\left(s_{1}, x_{2}\right)\right] \\
& +\left[f\left(s_{1}, x_{2}\right)-f\left(s_{1}, x_{1}\right)\right]=f^{\Delta}\left(s_{1}, x_{2}\right)\left(s_{2}-s_{1}\right) \\
& +\frac{\partial f}{\partial x}\left(s_{1}, x_{1}\right)\left(x_{2}-x_{1}\right)+\frac{1}{2} \frac{\partial^{2} f}{\partial x^{2}}\left(s_{1}, x_{1}\right)\left(x_{2}-x_{1}\right)^{2} \\
& +R_{\mathrm{TS}}^{f}\left(x_{2} ; s_{1}, s_{2}\right)+R_{\mathrm{C}}^{f}\left(s_{1} ; x_{1}, x_{2}\right)=\left[f^{\Delta}\left(s_{1}, x_{1}\right)\right. \\
& +\frac{\partial f^{\Delta}}{\partial x}\left(s_{1}, x_{1}\right)\left(x_{2}-x_{1}\right) \\
& \left.\quad+\frac{1}{2} \frac{\partial^{2} f^{\Delta}}{\partial x^{2}}\left(s_{1}, x_{1}\right)\left(x_{2}-x_{1}\right)^{2}+R_{\mathrm{C}}^{f^{\Delta}}\left(s_{1} ; x_{1}, x_{2}\right)\right]\left(s_{2}\right. \\
& \left.\quad-s_{1}\right)+\frac{\partial f}{\partial x}\left(s_{1}, x_{1}\right)\left(x_{2}-x_{1}\right)+\frac{1}{2} \frac{\partial^{2} f}{\partial x^{2}}\left(s_{1}, x_{1}\right)\left(x_{2}\right. \\
& \left.\quad-x_{1}\right)^{2}+R_{\mathrm{TS}}^{f}\left(x_{2} ; s_{1}, s_{2}\right)+R_{C}^{f}\left(s_{1} ; x_{1}, x_{2}\right) \\
& \quad=f^{\Delta}\left(s_{1}, x_{1}\right)\left(s_{2}-s_{1}\right)+\frac{\partial f}{\partial x}\left(s_{1}, x_{1}\right)\left(x_{2}-x_{1}\right)+\frac{1}{2} \\
& \quad \cdot \frac{\partial^{2} f}{\partial x^{2}}\left(s_{1}, x_{1}\right)\left(x_{2}-x_{1}\right)^{2}+R\left(s_{1}, s_{2} ; x_{1}, x_{2}\right),
\end{aligned}
$$

with

$$
\begin{aligned}
\left|R\left(s_{1}, s_{2} ; x_{1}, x_{2}\right)\right| \leq & r\left(\left|s_{2}-s_{1}\right|\right)\left|s_{2}-s_{1}\right| \\
& +r\left(\left|x_{2}-x_{1}\right|\right)\left(x_{2}-x_{1}\right)^{2}
\end{aligned}
$$

for another function $r: \mathbb{R}_{+} \rightarrow \mathbb{R}_{+}$increasing with $\lim _{u \downarrow 0} r(u)=0$.
Consider a partition $\pi^{(n)}: t_{1}=s_{0}<s_{1}<\cdots<s_{n}=t_{2}$, such that (1) each $s_{i} \in \mathbb{T}$; (2) $\max _{i}\left(\rho\left(s_{i}\right)-s_{i-1}\right) \leq 1 / 2^{n}$ for $i=1,2, \ldots, n$. Notice that by definition $\rho\left(s_{i}\right)=\sup \left\{s<s_{i}\right.$ : $s \in \mathbb{T}\}$, so that we can always find $s_{i-1} \in \mathbb{T}$ so that $\rho\left(s_{i}\right)-s_{i-1}$ is sufficiently small.

Let the sets $C$ and $\mathscr{I}$ be defined as before. Let us fix a partition $\pi^{(n)}$, and consider a classification of its corresponding intervals $\left(s_{i-1}, s_{i}\right), i=1,2, \ldots, n$. We will classify all intervals $\left(s_{i-1}, s_{i}\right)$ such that for all $I_{k} \in \mathscr{I}$ we have $I_{k} \cap\left(s_{i-1}, s_{i}\right)=\emptyset$ as class $(a)$; and we classify all intervals $\left(s_{i-1}, s_{i}\right)$ such that there exist some $I_{k} \in \mathscr{I}$ with $\left(s_{i-1}, s_{i}\right) \cap I_{k} \neq \emptyset$ as class (b). For an interval $\left(s_{i-1}, s_{i}\right)$ in class $(a)$, since for all $I_{k} \in \mathscr{I}$ we have $I_{k} \cap\left(s_{i-1}, s_{i}\right)=\emptyset$, we see that $\rho\left(s_{i}\right)=s_{i}$, because otherwise $\left(\rho\left(s_{i}\right), s_{i}\right)$ will be one of the $I_{k}$ 's. Thus in this case we have $s_{i}-s_{i-1}<1 / 2^{n}$. For an interval $\left(s_{i-1}, s_{i}\right)$ in class $(b)$, since both $s_{i-1}$ and $s_{i}$ are in $\mathbb{T}$, we see that we have in fact $I_{k} \subseteq\left(s_{i-1}, s_{i}\right)$. In this case either $I_{k}=\left(s_{i-1}, s_{i}\right)$, or $I_{k} \neq\left(s_{i-1}, s_{i}\right)$. If the latter happens, then $\left(\rho\left(s_{i}\right), s_{i}\right) \in \mathscr{I}$ is one of the $I_{k}$ 's and $\rho\left(s_{i}\right)-s_{i-1}<1 / 2^{n}$. We also see from the above analysis that all $I_{k}$ 's are contained in intervals $\left(s_{i-1}, s_{i}\right)$ that belong to class (b). On the other hand, either each interval $\left(s_{i-1}, s_{i}\right)$ is entirely one of the $I_{k}$ 's, or it contains an interval $\left(\rho\left(s_{i}\right), s_{i}\right)$ that is one of the $I_{k}$ 's. For the latter case, that is, when $s_{i-1}<\rho\left(s_{i}\right)<s_{i}$, the set of intervals of the form $\left(s_{i-1}, \rho\left(s_{i}\right)\right)$ are disjoint open intervals such that

$$
\sum_{\left(s_{i-1}, s_{i}\right) \in(b), s_{i-1}<\rho\left(s_{i}\right)<s_{i}}\left(\rho\left(s_{i}\right)-s_{i-1}\right)<\frac{n}{2^{n}} .
$$

Now we have

$$
\begin{aligned}
& f(\left.t_{2}, W_{t_{2}}\right)-f\left(t_{1}, W_{t_{1}}\right) \\
&= \sum_{i=1}^{n}\left[f\left(s_{i}, W_{s_{i}}\right)-f\left(s_{i-1}, W_{s_{i-1}}\right)\right] \\
&= \sum_{(a)}\left[f\left(s_{i}, W_{s_{i}}\right)-f\left(s_{i-1}, W_{s_{i-1}}\right)\right] \\
& \quad+\sum_{(b)}\left[f\left(s_{i}, W_{s_{i}}\right)-f\left(s_{i-1}, W_{s_{i-1}}\right)\right]=(I)+(I I) .
\end{aligned}
$$

We apply (19) term by term in part (I) of (22), and we get

$$
\begin{aligned}
(I) & =\sum_{(a)}\left[f\left(s_{i}, W_{s_{i}}\right)-f\left(s_{i-1}, W_{s_{i-1}}\right)\right] \\
& =\sum_{(a)}\left[f^{\Delta}\left(s_{i-1}, W_{s_{i-1}}\right)\left(s_{i}-s_{i-1}\right)\right. \\
& +\frac{\partial f}{\partial x}\left(s_{i-1}, W_{s_{i-1}}\right)\left(W_{s_{i}}-W_{s_{i-1}}\right) \\
& +\frac{1}{2} \frac{\partial^{2} f}{\partial x^{2}}\left(s_{i-1}, W_{s_{i-1}}\right)\left(W_{s_{i}}-W_{s_{i-1}}\right)^{2}
\end{aligned}
$$




$$
\begin{aligned}
& \left.+R\left(s_{i-1}, s_{i} ; W_{s_{i-1}}, W_{s_{i}}\right)\right] \\
& =\sum_{i=1}^{n}\left[f^{\Delta}\left(s_{i-1}, W_{s_{i-1}}\right)\left(s_{i}-s_{i-1}\right)\right. \\
& \left.+\frac{\partial f}{\partial x}\left(s_{i-1}, W_{s_{i-1}}\right)\left(W_{s_{i}}-W_{s_{i-1}}\right)\right] \\
& +\left(\sum_{(a)} \frac{1}{2} \frac{\partial^{2} f}{\partial x^{2}}\left(s_{i-1}, W_{s_{i-1}}\right)\left(W_{s_{i}}-W_{s_{i-1}}\right)^{2}\right. \\
& \left.+\sum_{(b)} \frac{1}{2} \frac{\partial^{2} f}{\partial x^{2}}\left(s_{i-1}, W_{s_{i-1}}\right)\left(s_{i}-s_{i-1}\right)\right) \\
& -\sum_{(b)}\left[f^{\Delta}\left(s_{i-1}, W_{s_{i-1}}\right)\left(s_{i}-s_{i-1}\right)\right. \\
& +\frac{\partial f}{\partial x}\left(s_{i-1}, W_{s_{i-1}}\right)\left(W_{s_{i}}-W_{s_{i-1}}\right) \\
& +\sum_{(a)} R\left(s_{i-1}, s_{i} ; W_{s_{i-1}}, W_{s_{i}}\right)=(I I I)_{1}+(I I I)_{2}+(I V) \\
& \left.+\frac{1}{2} \frac{\partial^{2} f}{\partial x^{2}}\left(s_{i-1}, W_{s_{i-1}}\right)\left(s_{i}-s_{i-1}\right)\right] \\
& +(V)
\end{aligned}
$$

We have the following four convergence results.

Convergence Result 1.1. By Lemma 6 ((35) and (36)) established below we have

$$
\begin{aligned}
& \mathbf{P}\left((I I I)_{1} \longrightarrow \int_{t_{1}}^{t_{2}} f^{\Delta}\left(s, W_{s}\right) \Delta s\right. \\
& \left.\quad+\int_{t_{1}}^{t_{2}} \frac{\partial f}{\partial x}\left(s, W_{s}\right) \Delta W_{s} \text { as } n \longrightarrow \infty\right)=1 .
\end{aligned}
$$

Convergence Result 1.2. By Lemma 7, (43), and Lemma 6, (35), established below we have

$$
\mathbf{P}\left((I I I)_{2} \longrightarrow \int_{t_{1}}^{t_{2}} \frac{1}{2} \frac{\partial^{2} f}{\partial x^{2}}\left(s, W_{s}\right) \Delta s \text { as } n \longrightarrow \infty\right)
$$$$
=1 \text {. }
$$

Convergence Result 2 . We have, with probability one, that

$$
(V)=\sum_{(a)} R\left(s_{i-1}, s_{i} ; W_{s_{i-1}}, W_{s_{i}}\right) \longrightarrow 0
$$

as $n \rightarrow \infty$.
In fact, by the Kolmogorov-Čentsov theorem proved in Theorem 3.1 of [5] we know that for almost all trajectories of $W_{t}$ on $\mathbb{T}$, for each fixed trajectory $W_{t}(\omega)$, there exists an $n_{0}=n_{0}(\omega)$ such that for all $n \geq n_{0}$, for a partition $\pi^{(n)}$ with a classification of its intervals $\left(s_{i-1}, s_{i}\right)$ into classes $(a)$ and $(b)$ as above, $\sup _{(a)}\left|W_{s_{i}}-W_{s_{i-1}}\right| \leq \delta / 2^{\gamma n / 5}$ for some fixed $\delta>0$ and $\gamma>0$. From here we can estimate

$$
\begin{aligned}
& \mathbf{E} \sum_{(a)} R\left(s_{i-1}, s_{i} ; W_{s_{i-1}}, W_{s_{i}}\right) \\
& \quad \leq \mathbf{E} \sum_{(a)}\left[r\left(s_{i}-s_{i-1}\right)\left(s_{i}-s_{i-1}\right)\right. \\
& \left.\quad+r\left(\left|W_{s_{i}}-W_{s_{i-1}}\right|\right)\left(W_{s_{i}}-W_{s_{i-1}}\right)^{2}\right] \leq r\left(\frac{1}{2^{n}}\right)\left(t_{2}\right. \\
& \left.\quad-t_{1}\right)+r\left(\frac{\delta}{2^{\text {rn/5 }}}\right)\left(t_{2}-t_{1}\right) ;
\end{aligned}
$$

that is,

$$
\mathbf{P}\left(\lim _{n \rightarrow \infty} \sum_{(a)} R\left(s_{i-1}, s_{i} ; W_{s_{i-1}}, W_{s_{i}}\right)=0\right)=1 .
$$

\section{Convergence Result 3. Let}

$$
\begin{aligned}
(I I) & +(I V)=A_{n}=A_{n}(\omega)=\sum_{(b)}\left[f\left(s_{i}, W_{s_{i}}\right)\right. \\
& -f\left(s_{i-1}, W_{s_{i-1}}\right)-f^{\Delta}\left(s_{i-1}, W_{s_{i-1}}\right)\left(s_{i}-s_{i-1}\right) \\
- & \frac{\partial f}{\partial x}\left(s_{i-1}, W_{s_{i-1}}\right)\left(W_{s_{i}}-W_{s_{i-1}}\right) \\
- & \left.\frac{1}{2} \frac{\partial^{2} f}{\partial x^{2}}\left(s_{i-1}, W_{s_{i-1}}\right)\left(s_{i}-s_{i-1}\right)\right], \\
B_{n}= & B_{n}(\omega)=\sum_{I_{k} \in \mathcal{F}, I_{k} \subset\left(t_{1}, t_{2}\right)}\left[f\left(s_{I_{k}}^{+}, W_{s_{I_{k}}^{+}}\right)\right. \\
- & f\left(s_{I_{k}}^{-}, W_{s_{I_{k}}^{-}}\right)-f^{\Delta}\left(s_{I_{k}}^{-}, W_{s_{I_{k}}^{-}}\right)\left(s_{I_{k}}^{+}-s_{I_{k}}^{-}\right) \\
- & \frac{\partial f}{\partial x}\left(s_{I_{k}}^{-}, W_{s_{I_{k}}^{-}}\right)\left(W_{s_{I_{k}}^{+}}-W_{s_{I_{k}}^{-}}\right) \\
& \left.-\frac{1}{2} \frac{\partial^{2} f}{\partial x^{2}}\left(s_{I_{k}}^{-}, W_{s_{I_{k}}^{-}}\right)\left(s_{I_{k}}^{+}-s_{I_{k}}^{-}\right)\right] .
\end{aligned}
$$

We claim that we have

$$
\mathbf{P}\left(\left|A_{n}(\omega)-B_{n}(\omega)\right| \longrightarrow 0 \text { as } n \longrightarrow \infty\right)=1 .
$$


In fact, from the analysis that leads to estimate (17) we see that we can write $A_{n}-B_{n}$ as

$$
\begin{aligned}
& A_{n}-B_{n}=\sum_{\left(s_{i-1}, s_{i}\right) \in(b), s_{i-1}<\rho\left(s_{i}\right)<s_{i}}\left[f\left(s_{i}, W_{s_{i}}\right)\right. \\
& -f\left(s_{i-1}, W_{s_{i-1}}\right)-f^{\Delta}\left(s_{i-1}, W_{s_{i-1}}\right)\left(s_{i}-s_{i-1}\right) \\
& -\frac{\partial f}{\partial x}\left(s_{i-1}, W_{s_{i-1}}\right)\left(W_{s_{i}}-W_{s_{i-1}}\right) \\
& \left.-\frac{1}{2} \frac{\partial^{2} f}{\partial x^{2}}\left(s_{i-1}, W_{s_{i-1}}\right)\left(s_{i}-s_{i-1}\right)\right] \\
& -\sum_{\left(s_{i-1}, s_{i}\right) \in(b), s_{i-1}<\rho\left(s_{i}\right)<s_{i}}\left[f\left(s_{i}, W_{s_{i}}\right)\right. \\
& -f\left(\rho\left(s_{i}\right), W_{\rho\left(s_{i}\right)}\right) \\
& -f^{\Delta}\left(\rho\left(s_{i}\right), W_{\rho\left(s_{i}\right)}\right)\left(s_{i}-\rho\left(s_{i}\right)\right) \\
& -\frac{\partial f}{\partial x}\left(\rho\left(s_{i}\right), W_{\rho\left(s_{i}\right)}\right)\left(W_{s_{i}}-W_{\rho\left(s_{i}\right)}\right) \\
& \left.-\frac{1}{2} \frac{\partial^{2} f}{\partial x^{2}}\left(\rho\left(s_{i}\right), W_{\rho\left(s_{i}\right)}\right)\left(s_{i}-\rho\left(s_{i}\right)\right)\right] \\
& -\sum_{I_{k} \in \mathscr{Y}, I_{k} \subset\left(s_{i-1}, \rho\left(s_{i}\right)\right) \text { for some }\left(s_{i-1}, s_{i}\right) \in(b)}\left[f\left(s_{I_{k}}^{+}, W_{s_{I_{k}}^{+}}\right)\right. \\
& -f\left(s_{I_{k}}^{-}, W_{s_{I_{k}}^{-}}\right)-f^{\Delta}\left(s_{I_{k}}^{-}, W_{s_{I_{k}}^{-}}\right)\left(s_{I_{k}}^{+}-s_{I_{k}}^{-}\right) \\
& -\frac{\partial f}{\partial x}\left(s_{I_{k}}^{-}, W_{s_{I_{k}}^{-}}\right)\left(W_{s_{I_{k}}^{+}}-W_{s_{I_{k}}^{-}}\right) \\
& \left.-\frac{1}{2} \frac{\partial^{2} f}{\partial x^{2}}\left(s_{I_{k}}^{-}, W_{s_{I_{k}}^{-}}\right)\left(s_{I_{k}}^{+}-s_{I_{k}}^{-}\right)\right]=(V I)_{1}+(V I)_{2} \\
& +(V I)_{3}+(V I)_{4}-(V I I) .
\end{aligned}
$$

Here

$$
\begin{aligned}
& (V I)_{1}=\sum_{\left(s_{i-1}, s_{i}\right) \in(b), s_{i-1}<\rho\left(s_{i}\right)<s_{i}}\left[f\left(\rho\left(s_{i}\right), W_{\rho\left(s_{i}\right)}\right)\right. \\
& \left.-f\left(s_{i-1}, W_{s_{i-1}}\right)\right] \text {, } \\
& (V I)_{2}=\sum_{\left(s_{i-1}, s_{i}\right) \in(b), s_{i-1}<\rho\left(s_{i}\right)<s_{i}}\left[f ^ { \Delta } ( \rho ( s _ { i } ) , W _ { \rho ( s _ { i } ) } ) \left(s_{i}\right.\right. \\
& \left.\left.-\rho\left(s_{i}\right)\right)-f^{\Delta}\left(s_{i-1}, W_{s_{i-1}}\right)\left(s_{i}-s_{i-1}\right)\right] \\
& =\sum_{\left(s_{i-1}, s_{i}\right) \in(b), s_{i-1}<\rho\left(s_{i}\right)<s_{i}}\left[\left(f^{\Delta}\left(\rho\left(s_{i}\right), W_{\rho\left(s_{i}\right)}\right)\right.\right. \\
& \left.-f^{\Delta}\left(s_{i-1}, W_{s_{i-1}}\right)\right)\left(s_{i}-s_{i-1}\right)-f^{\Delta}\left(\rho\left(s_{i}\right), W_{\rho\left(s_{i}\right)}\right) \\
& \left.\cdot\left(\rho\left(s_{i}\right)-s_{i-1}\right)\right] \text {, }
\end{aligned}
$$

$$
\begin{aligned}
& (V I)_{3}=\sum_{\left(s_{i-1}, s_{i}\right) \in(b), s_{i-1}<\rho\left(s_{i}\right)<s_{i}}\left[f ^ { \Delta } ( \rho ( s _ { i } ) , W _ { \rho ( s _ { i } ) } ) \left(W_{s_{i}}\right.\right. \\
& \left.\left.-W_{\rho\left(s_{i}\right)}\right)-f^{\Delta}\left(s_{i-1}, W_{s_{i-1}}\right)\left(W_{s_{i}}-W_{s_{i-1}}\right)\right] \\
& =\sum_{\left(s_{i-1}, s_{i}\right) \in(b), s_{i-1}<\rho\left(s_{i}\right)<s_{i}}\left[\left(f^{\Delta}\left(\rho\left(s_{i}\right), W_{\rho\left(s_{i}\right)}\right)\right.\right. \\
& \left.-f^{\Delta}\left(s_{i-1}, W_{s_{i-1}}\right)\right)\left(W_{s_{i}}-W_{s_{i-1}}\right) \\
& \left.-f^{\Delta}\left(\rho\left(s_{i}\right), W_{\rho\left(s_{i}\right)}\right)\left(W_{\rho\left(s_{i}\right)}-W_{s_{i-1}}\right)\right], \\
& (V I)_{4}=\sum_{\left(s_{i-1}, s_{i}\right) \in(b), s_{i-1}<\rho\left(s_{i}\right)<s_{i}}\left[f ^ { \Delta } ( \rho ( s _ { i } ) , W _ { \rho ( s _ { i } ) } ) \left(s_{i}\right.\right. \\
& \left.\left.-\rho\left(s_{i}\right)\right)-f^{\Delta}\left(s_{i-1}, W_{s_{i-1}}\right)\left(s_{i}-s_{i-1}\right)\right] \\
& =\sum_{\left(s_{i-1}, s_{i}\right) \in(b), s_{i-1}<\rho\left(s_{i}\right)<s_{i}}\left[f^{\Delta}\left(s_{i-1}, W_{s_{i-1}}\right)\left(s_{i-1}-\rho\left(s_{i}\right)\right)\right. \\
& +\left(f^{\Delta}\left(\rho\left(s_{i}\right), W_{\rho\left(s_{i}\right)}\right)-f^{\Delta}\left(s_{i-1}, W_{s_{i-1}}\right)\right)\left(s_{i}\right. \\
& \left.\left.-\rho\left(s_{i}\right)\right)\right] \text {, }
\end{aligned}
$$

$$
\begin{aligned}
& (V I I)=\sum_{I_{k} \in \mathcal{F}, I_{k} \subset\left(s_{i-1}, \rho\left(s_{i}\right)\right) \text { for some }\left(s_{i-1}, s_{i}\right) \in(b)}\left[f\left(s_{I_{k}}^{+}, W_{s_{I_{k}}^{+}}\right)\right. \\
& -f\left(s_{I_{k}}^{-}, W_{s_{I_{k}}^{-}}\right)-f^{\Delta}\left(s_{I_{k}}^{-}, W_{s_{I_{k}}^{-}}\right)\left(s_{I_{k}}^{+}-s_{I_{k}}^{-}\right) \\
& -\frac{\partial f}{\partial x}\left(s_{I_{k}}^{-}, W_{s_{I_{k}}^{-}}\right)\left(W_{s_{I_{k}}^{+}}-W_{s_{I_{k}}^{-}}\right)-\frac{1}{2} \frac{\partial^{2} f}{\partial x^{2}}\left(s_{I_{k}}^{-}, W_{s_{I_{k}}^{-}}\right) \\
& \left.\cdot\left(s_{I_{k}}^{+}-s_{I_{k}}^{-}\right)\right] .
\end{aligned}
$$

From (21), the Kolmogorov-Čentsov theorem proved in Theorem 3.1 of [5], as well as the assumptions about function $f$, we see that

$$
\begin{aligned}
& \mathbf{P}\left(\left|(V I)_{1}\right|+\left|(V I)_{2}\right|+\left|(V I)_{3}\right|+\left|(V I)_{4}\right|+|(V I I)|\right. \\
& \quad \longrightarrow 0 \text { as } n \longrightarrow \infty)=1 .
\end{aligned}
$$

From here we immediately see the claim (30).

Note that for any interval $I_{k}=\left(s_{I_{k}}^{-}, s_{I_{k}}^{+}\right)$we have $f^{\Delta}\left(s_{I_{k}}^{-}, W_{s_{I_{k}}^{-}}\right)=\left(f\left(s_{I_{k}}^{+}, W_{s_{I_{k}}^{-}}\right)-f\left(s_{I_{k}^{-}}, W_{s_{I_{k}}^{-}}\right)\right) /\left(s_{I_{k}}^{+}-s_{I_{k}}^{-}\right)$; therefore we see that

$$
\begin{aligned}
B_{n} & =B_{n}(\omega)=\sum_{I_{k} \in \mathcal{F}, I_{k} \subset\left(t_{1}, t_{2}\right)}\left[f\left(s_{I_{k}}^{+}, W_{s_{I_{k}}^{+}}\right)\right. \\
& -f\left(s_{I_{k}}^{-}, W_{s_{I_{k}}^{-}}\right)-\left[f\left(s_{I_{k}}^{+}, W_{s_{I_{k}}^{-}}\right)-f\left(s_{I_{k}^{-}}, W_{s_{I_{k}}^{-}}\right)\right] \\
& -\frac{\partial f}{\partial x}\left(s_{I_{k}}^{-}, W_{s_{I_{k}}^{-}}\right)\left(W_{s_{I_{k}}^{+}}-W_{s_{I_{k}}^{-}}\right)
\end{aligned}
$$




$$
\begin{aligned}
& \left.-\frac{1}{2} \frac{\partial^{2} f}{\partial x^{2}}\left(s_{I_{k}}^{-}, W_{s_{I_{k}}^{-}}\right)\left(s_{I_{k}}^{+}-s_{I_{k}}^{-}\right)\right] \\
& =\sum_{I_{k} \in \mathcal{F}, I_{k} c\left(t_{1}, t_{2}\right)}\left[f\left(s_{I_{k}}^{+}, W_{s_{I_{k}}^{+}}\right)-f\left(s_{I_{k}}^{+}, W_{s_{I_{k}}^{-}}\right)\right. \\
& -\frac{\partial f}{\partial x}\left(s_{I_{k}}^{-}, W_{s_{I_{k}}^{-}}\right)\left(W_{s_{I_{k}}^{+}}-W_{s_{I_{k}}^{-}}\right) \\
& \left.-\frac{1}{2} \frac{\partial^{2} f}{\partial x^{2}}\left(s_{I_{k}}^{-}, W_{s_{I_{k}}^{-}}\right)\left(s_{I_{k}}^{+}-s_{I_{k}}^{-}\right)\right] .
\end{aligned}
$$

Combining the convergence results (24), (25), (28), and (30), together with (22) and (23) and (34), we establish (14).

The next two lemmas are used in the above proof of Itô's formula, but they are also of independent interest.

Lemma 6 (convergence of $\Delta$-deterministic and stochastic integrals). Given a time scale $\mathbb{T}$ and $t_{1}, t_{2} \in \mathbb{T}, t_{1}<t_{2} ; a$ probability space $(\Omega, \mathscr{F}, \mathbf{P})$; a Brownian motion $\left\{W_{t}\right\}_{t \in \mathbb{T}}$ on the time scale $\mathbb{T}$, for any progressively measurable random function $f$ that is continuous on $\left[t_{1}, t_{2}\right] \cap \mathbb{T}$, viewed as a $L^{2}\left(\left[t_{1}, t_{2}\right]_{\mathbb{T}}\right)$ progressively measurable random function $f(t, \omega)$ on $\mathbb{T}$, and the families of partitions $\pi^{(n)}: t_{1}=s_{0}<s_{1}<\cdots<s_{n}=t_{2}$, $s_{0}, s_{1}, \ldots, s_{n} \in \mathbb{T}, \max _{i=1,2, \ldots, n}\left(\rho\left(s_{i}\right)-s_{i-1}\right)<1 / 2^{n}$, one has

$$
\begin{aligned}
& \mathbf{P}\left(\lim _{n \rightarrow \infty} \sum_{i=1}^{n} f\left(s_{i-1}, \omega\right)\left(s_{i}-s_{i-1}\right)=\int_{t_{1}}^{t_{2}} f(s, \omega) \Delta s\right) \\
& =1 \\
& \mathbf{P}\left(\lim _{n \rightarrow \infty} \sum_{i=1}^{n} f\left(s_{i-1}, \omega\right)\left(W_{s_{i}}-W_{s_{i-1}}\right)\right. \\
& \left.=\int_{t_{1}}^{t_{2}} f(s, \omega) \Delta W_{s}\right)=1 .
\end{aligned}
$$

Proof. As we have seen in the proof of Itô's formula, for a given partition $\pi^{(n)}: t_{1}=s_{0}<s_{1}<\cdots<s_{n}=t_{2}$, such that $s_{i} \in \mathbb{T}$ for $i=0,1, \ldots, n$, and $\max _{i=1,2, \ldots, n}\left(\rho\left(s_{i}\right)-s_{i-1}\right)<1 / 2^{n}$, we can classify all intervals of the form $\left(s_{i-1}, s_{i}\right)$ into two classes $(a)$ and $(b)$ : class $(a)$ is those open intervals $\left(s_{i-1}, s_{i}\right)$ such that it does not contain any open intervals $I_{k} \in \mathscr{I}$; class (b) is those open intervals $\left(s_{i-1}, s_{i}\right)$ such that it contains at least one open interval $I_{k} \in \mathscr{I}$, the latter of which has endpoints that are left- or right-scattered.

Let us form a family of partitions $\sigma^{(n)}: t_{1}=r_{0}<r_{1}<$ $\cdots<r_{m}=t_{2}$, so that the partition $\sigma^{(n)}$ is the partition $\pi^{(n)}$ together with all points in $\mathbb{T}$ that are of the form $r_{j}=\rho\left(s_{i}\right)$ for some $s_{i}$ in the partition $\pi^{(n)}$. Note that under this construction we have $r_{0}, r_{1}, \ldots, r_{m} \in \mathbb{T}$. In fact, for any interval $\left(s_{i-1}, s_{i}\right)$ in $(a)$, there is an identical interval $\left(r_{j-1}, r_{j}\right)$ in the partition $\sigma^{(n)}$ corresponding to it; for any interval $\left(s_{i-1}, s_{i}\right)$ in $(b)$, there are two intervals $\left(r_{j-2}, r_{j-1}\right)$ and $\left(r_{j-1}, r_{j}\right)$ corresponding to it, so that $r_{j-1}=\rho\left(s_{i}\right)$. And by (21) we know that

$$
\sum_{\left(s_{i-1}, s_{i}\right) \in(b),\left(r_{j-2}, r_{j-1}\right) \text { is corresponding to it }}\left(r_{j-1}-r_{j-2}\right)<\frac{n}{2^{n}} .
$$

Note that the number $m$ depends on $n$ and the partition $\pi^{(n)}: m=m\left(n, \pi^{(n)}\right)$. In particular $m \rightarrow \infty$ as $n \rightarrow \infty$. For simplicity we will suppress this dependence later in our proof.

Let us recall the definition of deterministic and stochastic $\Delta$-integrals as defined in Section 2. Let $\widetilde{f}$ be the extension of $f$ that we have in (8): for any $t \in \mathbb{T}$,

$$
\tilde{f}(t, \omega)=f\left(\sup [0, t]_{\mathbb{T}}, \omega\right) .
$$

Note that if $t \in \mathbb{T}$ is such that $\rho(t)=t$, then $\widetilde{f}(t, \omega)=f(t, \omega)$; otherwise if $t \in \mathbb{T}$ is such that $\rho(t)<t$, then $\tilde{f}(t, \omega)=$ $f(\rho(t), \omega)$. Thus we see that

$$
\begin{aligned}
& \mathbf{P}\left(\lim _{n \rightarrow \infty} \sum_{j=1}^{m} f\left(r_{j-1}, \omega\right)\left(r_{j}-r_{j-1}\right)=\int_{t_{1}}^{t_{2}} \tilde{f}(s, \omega) d s\right) \\
& =1 \\
& \mathbf{P}\left(\lim _{n \rightarrow \infty} \sum_{j=1}^{m} f\left(r_{j-1}, \omega\right)\left(W_{r_{j}}-W_{r_{j-1}}\right)\right. \\
& \left.=\int_{t_{1}}^{t_{2}} \tilde{f}(s, \omega) d W_{s}\right)=1 .
\end{aligned}
$$

So it suffices to prove that

$$
\begin{gathered}
\mathbf{P}\left(\operatorname { l i m } _ { n \rightarrow \infty } \left[\sum_{i=1}^{n} f\left(s_{i-1}, \omega\right)\left(s_{i}-s_{i-1}\right)\right.\right. \\
\left.\left.-\sum_{j=1}^{m} f\left(r_{j-1}, \omega\right)\left(r_{j}-r_{j-1}\right)\right]=0\right)=1, \\
\mathbf{P}\left(\operatorname { l i m } _ { n \rightarrow \infty } \left[\sum_{i=1}^{n} f\left(s_{i-1}, \omega\right)\left(W_{s_{i}}-W_{s_{i-1}}\right)\right.\right. \\
\left.\left.\quad-\sum_{j=1}^{m} f\left(r_{j-1}, \omega\right)\left(W_{r_{j}}-W_{r_{j-1}}\right)\right]=0\right)=1 .
\end{gathered}
$$

In fact, for any interval $\left(s_{i-1}, s_{i}\right)$ in class $(a)$, there exist an interval $\left(r_{j-1}, r_{j}\right)$ identical to the interval $\left(s_{i-1}, s_{i}\right)$, so that

$$
\begin{aligned}
& f\left(s_{i-1}, \omega\right)\left(s_{i}-s_{i-1}\right)-f\left(r_{j-1}, \omega\right)\left(r_{j}-r_{j-1}\right)=0, \\
& f\left(s_{i-1}, \omega\right)\left(W_{s_{i}}-W_{s_{i-1}}\right)-f\left(r_{j-1}, \omega\right)\left(W_{r_{j}}-W_{r_{j-1}}\right) \\
& \quad=0 .
\end{aligned}
$$


For any open interval $\left(s_{i-1}, s_{i}\right)$ in class $(b)$, there are two corresponding intervals $\left(r_{j-2}, r_{j-1}\right)$ and $\left(r_{j-1}, r_{j}\right)$ such that $r_{j-2}=s_{i-1}, r_{j-1}=\rho\left(s_{i}\right)$, and $r_{j}=s_{i}$. In this case

$$
\begin{aligned}
& f\left(s_{i-1}, \omega\right)\left(s_{i}-s_{i-1}\right)-f\left(r_{j-1}, \omega\right)\left(r_{j}-r_{j-1}\right) \\
&-f\left(r_{j-2}, \omega\right)\left(r_{j-1}-r_{j-2}\right)=f\left(s_{i-1}, \omega\right)\left(s_{i}-s_{i-1}\right) \\
&-f\left(\rho\left(s_{i}\right), \omega\right)\left(s_{i}-\rho\left(s_{i}\right)\right) \\
&-f\left(s_{i-1}, \omega\right)\left(\rho\left(s_{i}\right)-s_{i-1}\right) \\
&=\left(f\left(s_{i-1}, \omega\right)-f\left(\rho\left(s_{i}\right), \omega\right)\right)\left(s_{i}-\rho\left(s_{i}\right)\right), \\
& f\left(s_{i-1}, \omega\right)\left(W_{s_{i}}-W_{s_{i-1}}\right)-f\left(r_{j-1}, \omega\right)\left(W_{r_{j}}-W_{r_{j-1}}\right) \\
& \quad-f\left(r_{j-2}, \omega\right)\left(W_{r_{j-1}}-W_{r_{j-2}}\right) \\
& \quad=f\left(s_{i-1}, \omega\right)\left(W_{s_{i}}-W_{s_{i-1}}\right) \\
& \quad-f\left(\rho\left(s_{i}\right), \omega\right)\left(W_{s_{i}}-W_{\rho\left(s_{i}\right)}\right) \\
& \quad-f\left(s_{i-1}, \omega\right)\left(W_{\rho\left(s_{i}\right)}-W_{s_{i-1}}\right) \\
& \quad=\left(f\left(s_{i-1}, \omega\right)-f\left(\rho\left(s_{i}\right), \omega\right)\right)\left(W_{s_{i}}-W_{\rho\left(s_{i}\right)}\right) .
\end{aligned}
$$

From the above calculations and the fact that we have (21) and that $f$ is continuous on $\left[t_{1}, t_{2}\right] \cap \mathbb{T}$, together with the fact that $s_{j-1}, \rho\left(s_{j}\right) \in \mathbb{T}, 0 \leq \rho\left(s_{j}\right)-s_{j-1} \leq 1 / 2^{n}$, we see the claim as follows.

Lemma 7 (convergence of quadratic variation of Brownian motion on time scale). Given a time scale $\mathbb{T}$ and $t_{1}, t_{2} \in \mathbb{T}$, $t_{1}<t_{2}$; a probability space $(\Omega, \mathscr{F}, \mathbf{P})$; a Brownian motion $\left\{W_{t}\right\}_{t \in \mathbb{T}}$ on the time scale $\mathbb{T}$, let any $L^{2}\left(\left[t_{1}, t_{2}\right]_{\mathbb{T}}\right)$-progressively measurable random function $f(t, \omega)$ on $\mathbb{T}$ be defined such that $\mathbf{E} f^{2}(t, \omega)$ is uniformly bounded on $\left[t_{1}, t_{2}\right]$. Consider the families of partitions $\pi^{(n)}: t_{1}=s_{0}<s_{1}<\cdots<s_{n}=t_{2}$, $s_{0}, s_{1}, \ldots, s_{n} \in \mathbb{T}, \max _{i=1,2, \ldots, n}\left(\rho\left(s_{i}\right)-s_{i-1}\right)<1 / 2^{n}$. One classifies all the intervals $\left(s_{i-1}, s_{i}\right), i=1,2, \ldots, n$ into two classes $(a)$ and (b) as before. Then one has

$$
\begin{aligned}
\mathbf{P}\left(\operatorname { l i m } _ { n \rightarrow \infty } \left[\sum_{(a)} f\left(s_{i-1}, \omega\right)\left(W_{s_{i}}-W_{s_{i-1}}\right)^{2}\right.\right. \\
\left.\left.\quad-\sum_{(a)} f\left(s_{i-1}, \omega\right)\left(s_{i}-s_{i-1}\right)\right]=0\right)=1 .
\end{aligned}
$$

Proof. We notice that for all intervals $\left(s_{i-1}, s_{i}\right) \in(a)$ we have $\rho\left(s_{i}\right)=s_{i-1}$ and thus $s_{i}-s_{i-1}<1 / 2^{n}$. Let us denote that

$$
\begin{aligned}
V_{n} & =\left[\sum_{(a)} f\left(s_{i-1}, \omega\right)\left(W_{s_{i}}-W_{s_{i-1}}\right)^{2}\right. \\
& \left.-\sum_{(a)} f\left(s_{i-1}, \omega\right)\left(s_{i}-s_{i-1}\right)\right] .
\end{aligned}
$$

Since $f(t, \omega)$ is progressively measurable, we see that $f\left(s_{i-1}, \omega\right)$ is independent of $W_{s_{i}}-W_{s_{i-1}}$. Thus

$$
\begin{aligned}
\mathbf{E} V_{n} & =\mathbf{E}\left[\sum_{(a)} f\left(s_{i-1}, \omega\right)\left(W_{s_{i}}-W_{s_{i-1}}\right)^{2}\right. \\
& \left.-\sum_{(a)} f\left(s_{i-1}, \omega\right)\left(s_{i}-s_{i-1}\right)\right] \\
& =\sum_{(a)} \mathbf{E} f\left(s_{i-1}, \omega\right)\left(s_{i}-s_{i-1}\right) \\
& -\sum_{(a)} \mathbf{E} f\left(s_{i-1}, \omega\right)\left(s_{i}-s_{i-1}\right)=0
\end{aligned}
$$

Furthermore

$$
\begin{aligned}
& \operatorname{var} V_{n}=\mathbf{E}\left[\sum_{(a)} f\left(s_{i-1}, \omega\right)\left(W_{s_{i}}-W_{s_{i-1}}\right)^{2}\right. \\
& \left.-\sum_{(a)} f\left(s_{i-1}, \omega\right)\left(s_{i}-s_{i-1}\right)\right]^{2}=\mathbf{E} \sum_{(a)} f\left(s_{i-1}, \omega\right) \\
& \cdot f\left(s_{j-1}, \omega\right)\left[\left(W_{s_{i}}-W_{s_{i-1}}\right)^{2}-\left(s_{i}-s_{i-1}\right)\right] \\
& \cdot\left[\left(W_{s_{j}}-W_{s_{j-1}}\right)^{2}-\left(s_{j}-s_{j-1}\right)\right]=\sum_{(a)} \mathbf{E} f\left(s_{i-1}, \omega\right) \\
& \cdot f\left(s_{j-1}, \omega\right)\left[\left(W_{s_{i}}-W_{s_{i-1}}\right)^{2}-\left(s_{i}-s_{i-1}\right)\right] \\
& \cdot\left[\left(W_{s_{j}}-W_{s_{j-1}}\right)^{2}-\left(s_{j}-s_{j-1}\right)\right] .
\end{aligned}
$$

If $i<j$, then $f\left(s_{i-1}, \omega\right) f\left(s_{j-1}, \omega\right)\left[\left(W_{s_{i}}-W_{s_{i-1}}\right)^{2}-\left(s_{i}-\right.\right.$ $\left.\left.s_{i-1}\right)\right]$ is independent of $\left[\left(W_{s_{j}}-W_{s_{j-1}}\right)^{2}-\left(s_{j}-s_{j-1}\right)\right]$, so we have $\mathbf{E} f\left(s_{i-1}, \omega\right) f\left(s_{j-1}, \omega\right)\left[\left(W_{s_{i}}-W_{s_{i-1}}\right)^{2}-\left(s_{i}-s_{i-1}\right)\right] \cdot\left[\left(W_{s_{j}}-\right.\right.$ $\left.\left.W_{s_{j-1}}\right)^{2}-\left(s_{j}-s_{j-1}\right)\right]=0$. Similarly, for $i>j$ we also have $\mathbf{E} f\left(s_{i-1}, \omega\right) f\left(s_{j-1}, \omega\right)\left[\left(W_{s_{i}}-W_{s_{i-1}}\right)^{2}-\left(s_{i}-s_{i-1}\right)\right] \cdot\left[\left(W_{s_{j}}-W_{s_{j-1}}\right)^{2}-\right.$ $\left.\left(s_{j}-s_{j-1}\right)\right]=0$. This implies that

$$
\begin{aligned}
& \operatorname{var} V_{n}=\sum_{(a)} \mathbf{E} f^{2}\left(s_{i-1}, \omega\right)\left[\left(W_{s_{i}}-W_{s_{i-1}}\right)^{2}\right. \\
& \left.-\left(s_{i}-s_{i-1}\right)\right]^{2}=\sum_{(a)} \mathbf{E} f^{2}\left(s_{i-1}, \omega\right) \mathbf{E}\left[\left(W_{s_{i}}-W_{s_{i-1}}\right)^{2}\right. \\
& \left.-\left(s_{i}-s_{i-1}\right)\right]^{2}=\sum_{(a)} \mathbf{E} f^{2}\left(s_{i-1}, \omega\right) \mathbf{E}\left[\left(W_{s_{i}}-W_{s_{i-1}}\right)^{4}\right. \\
& \left.\quad-2\left(W_{s_{i}}-W_{s_{i-1}}\right)^{2}\left(s_{i}-s_{i-1}\right)+\left(s_{i}-s_{i-1}\right)^{2}\right]
\end{aligned}
$$




$$
\begin{aligned}
& =\sum_{(a)} \mathbf{E} f^{2}\left(s_{i-1}, \omega\right)\left[3\left(s_{i}-s_{i-1}\right)^{2}-2\left(s_{i}-s_{i-1}\right)^{2}\right. \\
& \left.+\left(s_{i}-s_{i-1}\right)^{2}\right]=2 \sum_{(a)} \mathbf{E} f^{2}\left(s_{i-1}, \omega\right)\left(s_{i}-s_{i-1}\right)^{2} \\
& \leq \frac{1}{2^{n-1}}\left(\max _{s \in\left[t_{1}, t_{2}\right]} \mathbf{E} f^{2}(s, \omega)\right) \sum_{(a)}\left(s_{i}-s_{i-1}\right) \longrightarrow 0
\end{aligned}
$$

as $n \rightarrow \infty$. This together with the fact that $\mathbf{E} V_{n}=0$ for any $n$ implies claim (43) of the lemma.

The argument above leads us to an Itô formula for $f\left(t, W_{t}\right)$. Making use of the same methods, one can derive a more general Itô formula for the solution $X_{t}$ to the $\Delta$ stochastic differential equation (11). We will not repeat the proof, but we will claim the following theorem.

Theorem 8 (general Itô's formula). Let $X_{t}$ be the solution to the $\Delta$-stochastic differential equation (11). Let any function $f: \mathbb{T} \times \mathbb{R} \rightarrow \mathbb{R}$ be such that $f^{\Delta}(t, x), f^{\Delta^{2}}(t, x),(\partial f / \partial x)(t, x)$, $\left(\partial^{2} f / \partial x^{2}\right)(t, x),\left(\partial f^{\Delta} / \partial x\right)(t, x)$, and $\left(\partial^{2} f^{\Delta} / \partial x^{2}\right)(t, x)$ are continuous on $\mathbb{\mathbb { V }} \times \mathbb{R}$. For any $t_{1} \leq t_{2}, t_{1}, t_{2} \in[0, \infty)_{\mathbb{T}}$ one has

$$
\begin{aligned}
& f\left(t_{2}, X_{t_{2}}\right)-f\left(t_{1}, X_{t_{1}}\right)=\int_{t_{1}}^{t_{2}} b\left(s, X_{s}\right) f^{\Delta}\left(s, X_{s}\right) \Delta s \\
& +\int_{t_{1}}^{t_{2}} \sigma\left(s, X_{s}\right) \frac{\partial f}{\partial x}\left(s, X_{s}\right) \Delta W_{s}+\frac{1}{2} \\
& \quad \cdot \int_{t_{1}}^{t_{2}} \sigma^{2}\left(s, X_{s}\right) \frac{\partial^{2} f}{\partial x^{2}}\left(s, X_{s}\right) \Delta s \\
& +\sum_{I_{k} \in \mathcal{F}, I_{k} \subset\left(t_{1}, t_{2}\right)}\left[f\left(s_{I_{k}}^{+}, W_{s_{I_{k}}^{+}}\right)-f\left(s_{I_{k}}^{+}, W_{s_{I_{k}}^{-}}\right)-\sigma\left(s_{I_{k}}^{-},\right.\right. \\
& \left.W_{s_{I_{k}}^{-}}\right) \frac{\partial f}{\partial x}\left(s_{I_{k}}^{-}, W_{s_{I_{k}}^{-}}\right)\left(W_{s_{I_{k}}^{+}}-W_{s_{I_{k}}^{-}}\right) \\
& -\frac{1}{2} \sigma^{2}\left(s_{I_{k}}^{-},\right. \\
& \left.\left.W_{s_{I_{k}}^{-}}\right) \frac{\partial^{2} f}{\partial x^{2}}\left(s_{I_{k}}^{-}, W_{s_{I_{k}}^{-}}\right)\left(s_{I_{k}}^{+}-s_{I_{k}}^{-}\right)\right] .
\end{aligned}
$$

\section{The Stochastic Exponential on Time Scales}

Our target in this section is to establish a closed-form formula for the stochastic exponential in the case of general time scales T.

Definition 9. One says an adapted stochastic process $A(t)$ defined on the filtered probability space $\left(\Omega, \mathscr{F}_{t}, \mathbf{P}\right)$ is stochastic regressive with respect to the Brownian motion $W_{t}$ on the time scale $\mathbb{T}$ if and only if for any right-scattered point $t \in \mathbb{T}$ one has

$$
(1+A(t))\left(W_{\sigma(t)}-W_{t}\right) \neq 0, \quad \text { a.s. } \forall t \in \mathbb{T} .
$$

The set of stochastic regressive processes will be denoted by $\mathscr{R}_{W}$.
The following definition of a stochastic exponential was also introduced in [3].

Definition 10 (stochastic exponential). Let $t_{0} \in \mathbb{T}$ and $A \in$ $\mathscr{R}_{W}$; then the unique solution of the $\Delta$-stochastic differential equation

$$
\begin{aligned}
\Delta X_{t} & =A(t) X_{t} \Delta W_{t}, \\
X\left(t_{0}\right) & =1,
\end{aligned}
$$

$$
t \in \mathbb{T}
$$

is called the stochastic exponential and is denoted by

$$
X_{\bullet}=\mathscr{E}_{A}\left(\bullet, t_{0}\right) \text {. }
$$

We note that $\mathscr{E}_{A}\left(t, t_{0}\right)$ as a solution to (50) can be written into an integral equation

$$
\mathscr{E}_{A}\left(t, t_{0}\right)=1+\int_{t_{0}}^{t} A(s) \mathscr{E}_{A}\left(s, t_{0}\right) \Delta W_{s}, \quad \forall t \in \mathbb{T} .
$$

We will be making use of the set-up we have in Section 4 about Itô's formula. Let $t_{0}<t$ and $t_{0}, t \in \mathbb{T}$. Let the sets $C$ and $\mathscr{I}$ be defined as in Section 4 corresponding to the interval $\left[t_{1}, t_{2}\right]=\left[t_{0}, t\right]$. Let $I_{k} \in \mathscr{I}$ and $I_{k}=\left(s_{I_{k}}^{-}, s_{I_{k}}^{+}\right)$. We note that $s_{I_{k}}^{-}=\rho\left(s_{I_{k}}^{+}\right), s_{I_{k}}^{+}=\sigma\left(s_{I_{k}}^{-}\right)$. Let

$$
\begin{aligned}
D\left(t, t_{0}\right)= & \sum_{I_{k} \in \mathcal{F}, I_{k} \subset\left(t_{0}, t\right)} A\left(s_{I_{k}}^{-}\right)\left(W_{s_{I_{k}}^{+}}-W_{s_{I_{k}}^{-}}\right) \\
& -\frac{1}{2} \sum_{I_{k} \in \mathcal{F}, I_{k} \subset\left(t_{0}, t\right)} A^{2}\left(s_{I_{k}}^{-}\right)\left(s_{I_{k}}^{+}-s_{I_{k}}^{-}\right) .
\end{aligned}
$$

We define

$$
\begin{aligned}
& U\left(t, t_{0}\right)=\prod_{I_{k} \in \mathcal{F}, I_{k} \subset\left(t_{0}, t\right)}\left[1+A\left(s_{I_{k}}^{-}\right)\left(W_{s_{I_{k}}^{+}}-W_{s_{I_{k}}^{-}}\right)\right], \\
& V\left(t, t_{0}\right) \\
& \quad=\exp \left(\int_{t_{0}}^{t} A(s) \Delta W_{s}-\frac{1}{2} \int_{t_{0}}^{t} A^{2}(s) \Delta s-D\left(t, t_{0}\right)\right) .
\end{aligned}
$$

Theorem 11 (stochastic exponential on time scales). The stochastic exponential has the closed-form expression

$$
\mathscr{E}_{A}\left(t, t_{0}\right)=U\left(t, t_{0}\right) V\left(t, t_{0}\right)
$$

Proof. Consider the process

$$
Y_{t}=\int_{t_{0}}^{t} A(s) \Delta W_{s}-\frac{1}{2} \int_{t_{0}}^{t} A^{2}(s) \Delta s-D\left(t, t_{0}\right) .
$$

Let us introduce another function $\alpha(t)$ such that

$$
\alpha(t)= \begin{cases}0, & \text { when } t=s_{I_{k}}^{-} \text {or } t=s_{I_{k}}^{+}, \\ A(t), & \text { otherwise. }\end{cases}
$$


We see now that the process $Y_{t}$ is a solution to the $\Delta$ stochastic differential equation

$$
\begin{aligned}
\Delta Y_{t} & =\alpha(t) \Delta W_{s}-\frac{1}{2} \alpha^{2}(t) \Delta s \\
Y_{t_{0}} & =0
\end{aligned}
$$

Notice that $Y_{s_{I_{k}}^{-}}=Y_{s_{I_{k}}^{+}}$for any $I_{k}=\left(s_{I_{k}}^{-}, s_{I_{k}}^{+}\right) \in \mathscr{I}$. Taking this into account, as well as the fact that $\alpha(s)=0$ whenever $t=s_{I_{k}}^{-}$or $t=s_{I_{k}}^{+}$, we can apply the general Itô formula (48) to the function $V\left(t, t_{0}\right)=\exp \left(Y_{t}\right)$ and we will get

$$
\begin{aligned}
\exp \left(Y_{t}\right)-1= & \int_{t_{0}}^{t} \alpha(s) \exp \left(Y_{s}\right) \Delta W_{s} \\
& -\frac{1}{2} \int_{t_{0}}^{t} \alpha^{2}(s) \exp \left(Y_{s}\right) \Delta s \\
& +\frac{1}{2} \int_{t_{0}}^{t} \alpha^{2}(s) \exp \left(Y_{s}\right) \Delta s \\
= & \int_{t_{0}}^{t} \alpha(s) \exp \left(Y_{s}\right) \Delta W_{s} .
\end{aligned}
$$

Thus

$$
V\left(t, t_{0}\right)=1+\int_{t_{0}}^{t} \alpha(s) V\left(s, t_{0}\right) \Delta W_{s},
$$

or in other words

$$
\Delta V\left(t, t_{0}\right)=\alpha(t) V\left(t, t_{0}\right) \Delta W_{t}
$$

Let us now consider the function $\mathscr{E}_{A}\left(t, t_{0}\right)=$ $U\left(t, t_{0}\right) V\left(t, t_{0}\right)$. We claim that

$$
\begin{aligned}
& U\left(t, t_{0}\right) V\left(t, t_{0}\right)-1 \\
& \quad=\int_{t_{0}}^{t} A(s) U\left(s, t_{0}\right) V\left(s, t_{0}\right) \Delta W_{s} .
\end{aligned}
$$

Notice that

$$
\begin{aligned}
U\left(s_{I_{k}}^{+}, t_{0}\right)= & {\left[1+A\left(s_{I_{k}}^{-}\right)\left(W_{s_{I_{k}}^{+}}-W_{s_{I_{k}}^{-}}\right)\right] U\left(s_{I_{k}}^{-}, t_{0}\right) } \\
= & U\left(s_{I_{k}}^{-}, t_{0}\right) \\
& +A\left(s_{I_{k}}^{-}\right) U\left(s_{I_{k}}^{-}, t_{0}\right)\left(W_{s_{I_{k}}^{+}}-W_{s_{I_{k}}^{-}}\right) ;
\end{aligned}
$$

that is,

$$
\begin{aligned}
& U\left(s_{I_{k}}^{+}, t_{0}\right)-U\left(s_{I_{k}}^{-}, t_{0}\right) \\
& \quad=A\left(s_{I_{k}}^{-}\right) U\left(s_{I_{k}}^{-}, t_{0}\right)\left(W_{s_{I_{k}}^{+}}-W_{s_{I_{k}}^{-}}\right) .
\end{aligned}
$$

Using this fact, the above claimed identity (62) can be written as

$$
\begin{gathered}
U\left(t, t_{0}\right) V\left(t, t_{0}\right)-1=\int_{t_{0}}^{t} \alpha(s) U\left(s, t_{0}\right) V\left(s, t_{0}\right) \Delta W_{s} \\
+\sum_{I_{k} \in \mathcal{F}, I_{k} \subset\left(t_{0}, t\right)} A\left(s_{I_{k}}^{-}\right) U\left(s_{I_{k}}^{-}, t_{0}\right) V\left(s_{I_{k}}^{-}, t_{0}\right) \\
\cdot\left(W_{s_{I_{k}}^{+}}-W_{s_{I_{k}}^{-}}\right)=\int_{t_{0}}^{t} \alpha(s) U\left(s, t_{0}\right) V\left(s, t_{0}\right) \Delta W_{s} \\
+\sum_{I_{k} \in \mathscr{F}, I_{k} \subset\left(t_{0}, t\right)} V\left(s_{I_{k}}^{-}, t_{0}\right)\left(U\left(s_{I_{k}}^{+}, t_{0}\right)-U\left(s_{I_{k}}^{-}, t_{0}\right)\right) .
\end{gathered}
$$

In fact, with respect to the partition $\pi^{(n)}: t_{0}=s_{0}<s_{1}<$ $\cdots<s_{n-1}<s_{n}=t$ that we have been using, we have

$$
\begin{aligned}
& U\left(t, t_{0}\right) V\left(t, t_{0}\right)-1=\sum_{i=1}^{n}\left[U\left(s_{i}, t_{0}\right) V\left(s_{i}, t_{0}\right)\right. \\
& \left.-U\left(s_{i-1}, t_{0}\right) V\left(s_{i-1}, t_{0}\right)\right] \\
& =\sum_{i=1}^{n}\left[\left(U\left(s_{i}, t_{0}\right)-U\left(s_{i-1}, t_{0}\right)\right)\right. \\
& \cdot\left(V\left(s_{i}, t_{0}\right)-V\left(s_{i-1}, t_{0}\right)\right)+U\left(s_{i-1}, t_{0}\right) \\
& \cdot\left(V\left(s_{i}, t_{0}\right)-V\left(s_{i-1}, t_{0}\right)\right)+V\left(s_{i-1}, t_{0}\right) \\
& \left.\cdot\left(U\left(s_{i}, t_{0}\right)-U\left(s_{i-1}, t_{0}\right)\right)\right]=\sum_{i=1}^{n}\left(U\left(s_{i}, t_{0}\right)\right. \\
& \left.-U\left(s_{i-1}, t_{0}\right)\right)\left(V\left(s_{i}, t_{0}\right)-V\left(s_{i-1}, t_{0}\right)\right) \\
& +\sum_{i=1}^{n} U\left(s_{i-1}, t_{0}\right)\left(V\left(s_{i}, t_{0}\right)-V\left(s_{i-1}, t_{0}\right)\right) \\
& +\sum_{i=1}^{n} V\left(s_{i-1}, t_{0}\right)\left(U\left(s_{i}, t_{0}\right)-U\left(s_{i-1}, t_{0}\right)\right)=(I) \\
& +(I I)+(I I I) .
\end{aligned}
$$

Here

$$
\begin{aligned}
& (I)=\sum_{i=1}^{n}\left(U\left(s_{i}, t_{0}\right)-U\left(s_{i-1}, t_{0}\right)\right) \\
& \cdot\left(V\left(s_{i}, t_{0}\right)-V\left(s_{i-1}, t_{0}\right)\right), \\
& (I I)=\sum_{i=1}^{n} U\left(s_{i-1}, t_{0}\right)\left(V\left(s_{i}, t_{0}\right)-V\left(s_{i-1}, t_{0}\right)\right), \\
& (I I I)=\sum_{i=1}^{n} V\left(s_{i-1}, t_{0}\right)\left(U\left(s_{i}, t_{0}\right)-U\left(s_{i-1}, t_{0}\right)\right) .
\end{aligned}
$$

We can apply the previous arguments and classify the intervals $\left(s_{i-1}, s_{i}\right)$ into classes $(a)$ and $(b)$. Notice that, on each interval $\left(s_{I_{k}}^{-}, s_{I_{k}}^{+}\right)$, the function $V\left(t, t_{0}\right)$ remains constant and the function $U\left(t, t_{0}\right)$ has a jump, and on each interval $\left(s_{i-1}, s_{i}\right)$ 
in class $(a)$ the function $U\left(t, t_{0}\right)$ is a constant. This observation and similar arguments (which we leave to the reader) as in the previous section will enable us to prove that, with probability one, as $n \rightarrow \infty$, we will have

$$
\begin{aligned}
& (I) \longrightarrow 0 \\
& (I I) \longrightarrow \int_{t_{0}}^{t} \alpha(s) U\left(s, t_{0}\right) V\left(s, t_{0}\right) \Delta W_{s},
\end{aligned}
$$

$$
\longrightarrow \sum_{I_{k} \in \mathcal{F}, I_{k} \subset\left(t_{0}, t\right)} V\left(s_{I_{k}}^{-}, t_{0}\right)\left(U\left(s_{I_{k}}^{+}, t_{0}\right)-U\left(s_{I_{k}}^{-}, t_{0}\right)\right) .
$$

So we proved (65) and thus (62).

\section{Change of Measure and Girsanov's Theorem on Time Scales}

We demonstrate in this section a change of measure formula (Girsanov's formula) for Brownian motion on time scales. Our analysis is based on the method of extension that was introduced in Section 3 (originally from [2]).

Let us consider two processes: the standard Brownian motion $\left\{W_{t}\right\}_{t \in \mathbb{T}}$ on $\left(\Omega, \mathscr{F}_{t}, \mathbf{P}\right)$ on the time scale $\mathbb{T}$ and the process

$$
B_{t}=W_{t}-\int_{0}^{t} A(s) \Delta s
$$

on the time scale $t \in \mathbb{T}$.

Let us consider an extension of the (probably random) function $A(s)$ as in (8). Let us define the so obtained extension function to be $\widetilde{A}(s)$. Recall that (8) implies that

$$
\widetilde{A}(s, \omega)=A\left(\sup [0, s]_{\mathbb{T}}, \omega\right) .
$$

Let $\widetilde{W}_{t}$ be a standard Brownian motion on $[0, \infty)$. If we define

$$
\widetilde{B}_{t}=\widetilde{W}_{t}-\int_{0}^{t} \widetilde{A}(s) d s,
$$

then the process $\widetilde{B}_{t}$ agrees with $B_{t}$ for any time point $t \in \mathbb{T}$.

For any $t, t_{0} \in \mathbb{T}, t>t_{0}$, let

$$
\begin{aligned}
\mathscr{G}_{A}\left(t, t_{0}\right) & =\exp \left(\int_{t_{0}}^{t} \widetilde{A}(s) d W_{s}-\frac{1}{2} \int_{t_{0}}^{t} \widetilde{A}^{2}(s) d s\right) \\
& =\exp \left(\int_{t_{0}}^{t} \widetilde{A}(s) d W_{s}-\frac{1}{2} \int_{t_{0}}^{t} \widetilde{A^{2}}(s) d s\right) \\
& =\exp \left(\int_{t_{0}}^{t} A(s) \Delta W_{s}-\frac{1}{2} \int_{t_{0}}^{t} A^{2}(s) \Delta s\right) .
\end{aligned}
$$

It is easy to see that the function $\mathscr{G}_{A}\left(t, t_{0}\right)$ is the standard Girsanov's density function for the process $\widetilde{B}_{t}$ with respect to the standard Brownian motion $\widetilde{W}_{t}$. Since $\widetilde{B}_{t}$ and $\widetilde{W}_{t}$ have the same distributions as $B_{t}$ and $W_{t}$ on the time scale $\mathbb{T}$, we conclude with the following two Theorems.
Theorem 12 (Novikov's condition on time scales). If for every $t \geq 0$ one has

$$
\mathbf{E} \exp \left(\int_{0}^{t} A^{2}(s) \Delta s\right)<\infty,
$$

then for every $t \geq 0$ one has

$$
\mathbf{E} \mathscr{G}_{A}\left(t, t_{0}\right)=1 \text {. }
$$

Let (73) be satisfied. Let $T>0$ and pick $T>t_{0}, t_{0}, T \in$ $\mathbb{T}$. Consider a new measure $\mathbf{P}^{B}$ on $\left(\Omega, \mathscr{F}_{t}\right)$, defining by it Radon-Nikodym derivative with respect to $\mathbf{P}^{W}$, as

$$
\frac{d \mathbf{P}^{B}}{d \mathbf{P}^{W}}=\mathscr{G}_{A}\left(T, t_{0}\right)
$$

Theorem 13 (Girsanov's change of measure on time scales). Under the measure $\mathbf{P}^{B}$ the process $B_{t}, t \in[0, T]_{\mathbb{T}}$, is a standard Brownian motion on $\mathbb{T}$.

\section{Application to Brownian Motion on a Quantum Time Scale}

In this section we are going to apply our result to a quantum time scale ( $q$-time scale, see [1, Example 1.41]). Let $q>1$ and

$$
\begin{aligned}
& q^{\mathbb{Z}}:=\left\{q^{k}: k \in \mathbb{Z}\right\}, \\
& \overline{q^{\mathbb{Z}}}:=q^{\mathbb{Z}} \cup\{0\} .
\end{aligned}
$$

The quantum time scale ( $q$-time scale) is defined by $\mathbb{T}=$ $\overline{q \mathbb{Z}}$. Given the quantum time scale $\mathbb{T}$, one can then construct a Brownian motion $W_{t}$ on $\mathbb{T}$ according to Definition 3 .

We have

$$
\sigma(t)=\inf \left\{q^{n}: n \in[m+1, \infty)\right\}=q^{m+1}=q q^{m}=q t
$$

if $t=q^{m} \in \mathbb{T}$ and obviously $\sigma(0)=0$. So we obtain

$$
\begin{aligned}
& \sigma(t)=q t, \\
& \rho(t)=\frac{t}{q},
\end{aligned}
$$

\section{$\forall t \in \mathbb{T}$}

and consequently

$$
\mu(t)=\sigma(t)-t=(q-1) t \quad \forall t \in \mathbb{T} .
$$

Here 0 is a right-dense minimum and every other point in $\mathbb{T}$ is isolated. For a function $f: \mathbb{T} \rightarrow \mathbb{R}$ we have

$$
f^{\Delta}(t)=\frac{f(\sigma(t))-f(t)}{\mu(t)}=\frac{f(q t)-f(t)}{(q-1) t}
$$

$$
f^{\Delta}(0)=\lim _{s \rightarrow 0} \frac{f(0)-f(s)}{0-s}=\lim _{s \rightarrow 0} \frac{f(s)-f(0)}{s}
$$

provided the limit exists. 
The open intervals $I_{k}$ that we have constructed in Section 4 have the form $I_{k}=\left(q^{k}, q^{k+1}\right)$ where $k \in \mathbb{Z}$. For any two points $t_{1}<t_{2}, t_{1}, t_{2} \in \mathbb{T}$, if $t_{1}, t_{2} \neq 0$, then $t_{1}=q^{k_{1}}$ and $t_{2}=q^{k_{2}}$ for two integers $k_{1}<k_{2}$. In this case we can apply (14) and we get

$$
\begin{aligned}
& f\left(q^{k_{2}}, W_{q^{k_{2}}}\right)-f\left(q^{k_{1}}, W_{q^{k_{1}}}\right)=\int_{q^{k_{1}}}^{q^{k_{2}}} f^{\Delta}\left(s, W_{s}\right) \Delta s \\
& \quad+\int_{q^{k_{1}}}^{q^{k_{2}}} \frac{\partial f}{\partial x}\left(s, W_{s}\right) \Delta W_{s}+\frac{1}{2} \int_{q^{k_{1}}}^{q^{k_{2}}} \frac{\partial^{2} f}{\partial x^{2}}\left(s, W_{s}\right) \Delta s \\
& \quad+\sum_{k=k_{1}}^{k_{2}-1}\left[f\left(q^{k+1}, W_{q^{k+1}}\right)-f\left(q^{k+1}, W_{q^{k}}\right)\right. \\
& -\frac{\partial f}{\partial x}\left(q^{k}, W_{q^{k}}\right)\left(W_{q^{k+1}}-W_{q^{k}}\right) \\
& \left.\quad-\frac{1}{2} \frac{\partial^{2} f}{\partial x^{2}}\left(q^{k}, W_{q^{k}}\right)\left(q^{k+1}-q^{k}\right)\right] .
\end{aligned}
$$

Since $\mathbb{T} \backslash\{0\}$ is a discrete time scale, we have

$$
\begin{aligned}
& \int_{q^{k_{1}}}^{q^{k_{2}}} \frac{\partial f}{\partial x}\left(s, W_{s}\right) \Delta W_{s} \\
& =\sum_{k=k_{1}}^{k_{2}-1} \frac{\partial f}{\partial x}\left(q^{k}, W_{q^{k}}\right)\left(W_{q^{k+1}}-W_{q^{k}}\right), \\
& \frac{1}{2} \int_{q^{k_{1}}}^{q^{k_{2}}} \frac{\partial^{2} f}{\partial x^{2}}\left(s, W_{s}\right) \Delta s \\
& =\sum_{k=k_{1}}^{k_{2}-1} \frac{1}{2} \frac{\partial^{2} f}{\partial x^{2}}\left(q^{k}, W_{q^{k}}\right)\left(q^{k+1}-q^{k}\right) .
\end{aligned}
$$

Moreover, we have

$$
\begin{aligned}
& \int_{q^{k_{1}}}^{q^{k_{2}}} f^{\Delta}\left(s, W_{s}\right) \Delta s \\
& =\sum_{k=k_{1}}^{k_{2}-1} \frac{f\left(q^{k+1}, W_{q^{k}}\right)-f\left(q^{k}, W_{q^{k}}\right)}{q^{k+1}-q^{k}}\left(q^{k+1}-q^{k}\right) \\
& =\sum_{k=k_{1}}^{k_{2}-1}\left[f\left(q^{k+1}, W_{q^{k}}\right)-f\left(q^{k}, W_{q^{k}}\right)\right] .
\end{aligned}
$$

Therefore (81) becomes

$$
\begin{aligned}
& f\left(q^{k_{2}}, W_{q^{k_{2}}}\right)-f\left(q^{k_{1}}, W_{q^{k_{1}}}\right) \\
& \quad=\sum_{k=k_{1}}^{k_{2}-1}\left\{\left[f\left(q^{k+1}, W_{q^{k}}\right)-f\left(q^{k}, W_{q^{k}}\right)\right]\right. \\
& \left.+\left[f\left(q^{k+1}, W_{q^{k+1}}\right)-f\left(q^{k+1}, W_{q^{k}}\right)\right]\right\} \\
& \quad=\sum_{k=k_{1}}^{k_{2}-1}\left[f\left(q^{k+1}, W_{q^{k+1}}\right)-f\left(q^{k}, W_{q^{k}}\right)\right],
\end{aligned}
$$

which is a trivial telescoping identity. This justifies (14) in the case away from 0.

Let us consider now the case when $t_{1}=0$ and $t_{2}=q^{k}>0$ for some $k \in \mathbb{Z}$. In this case we have, according to (14), that

$$
\begin{aligned}
& f\left(q^{k}, W_{q^{k}}\right)-f(0,0)=\int_{0}^{q^{k}} f^{\Delta}\left(s, W_{s}\right) \Delta s \\
& \quad+\int_{0}^{q^{k}} \frac{\partial f}{\partial x}\left(s, W_{s}\right) \Delta W_{s}+\frac{1}{2} \int_{0}^{q^{k}} \frac{\partial^{2} f}{\partial x^{2}}\left(s, W_{s}\right) \Delta s \\
& \quad+\sum_{j=-\infty}^{k-1}\left[f\left(q^{j+1}, W_{q^{j+1}}\right)-f\left(q^{j+1}, W_{q^{j}}\right)\right. \\
& \quad-\frac{\partial f}{\partial x}\left(q^{j}, W_{q^{j}}\right)\left(W_{q^{j+1}}-W_{q^{j}}\right) \\
& \left.\quad-\frac{1}{2} \frac{\partial^{2} f}{\partial x^{2}}\left(q^{j}, W_{q^{j}}\right)\left(q^{j+1}-q^{j}\right)\right] .
\end{aligned}
$$

One can justify that in this case we have

$$
\begin{aligned}
& \int_{0}^{q^{k}} \frac{\partial f}{\partial x}\left(s, W_{s}\right) \Delta W_{s} \\
& \quad=\sum_{j=-\infty}^{k-1} \frac{\partial f}{\partial x}\left(q^{j}, W_{q^{j}}\right)\left(W_{q^{j+1}}-W_{q^{j}}\right), \\
& \frac{1}{2} \int_{0}^{q^{k}} \frac{\partial^{2} f}{\partial x^{2}}\left(s, W_{s}\right) \Delta s \\
& \quad=\sum_{j=-\infty}^{k-1} \frac{1}{2} \frac{\partial^{2} f}{\partial x^{2}}\left(q^{j}, W_{q^{j}}\right)\left(q^{j+1}-q^{j}\right) .
\end{aligned}
$$

Moreover, we have

$$
\begin{aligned}
\int_{0}^{q^{k}} & f^{\Delta}\left(s, W_{s}\right) \Delta s \\
& =\sum_{j=-\infty}^{k-1} \frac{f\left(q^{j+1}, W_{q^{j}}\right)-f\left(q^{j}, W_{q^{j}}\right)}{q^{j+1}-q^{j}}\left(q^{j+1}-q^{j}\right) \\
& =\sum_{j=-\infty}^{k-1}\left[f\left(q^{j+1}, W_{q^{j}}\right)-f\left(q^{j}, W_{q^{j}}\right)\right] .
\end{aligned}
$$

Therefore (81) becomes

$$
\begin{aligned}
f & \left(q^{k}, W_{q^{k}}\right)-f(0,0) \\
& =\sum_{j=-\infty}^{k-1}\left\{\left[f\left(q^{j+1}, W_{q^{j}}\right)-f\left(q^{j}, W_{q^{j}}\right)\right]\right. \\
& \left.+\left[f\left(q^{j+1}, W_{q^{j+1}}\right)-f\left(q^{j+1}, W_{q^{j}}\right)\right]\right\} \\
& =\sum_{j=-\infty}^{k-1}\left[f\left(q^{j+1}, W_{q^{j+1}}\right)-f\left(q^{j}, W_{q^{j}}\right)\right],
\end{aligned}
$$


which is also a telescoping identity. This justifies (14) in the case including 0 .

Making use of Theorem 11, it is easy to write down the stochastic exponential for the quantum time scale:

$$
\mathscr{E}_{A}\left(0, q^{k}\right)=\prod_{j=-\infty}^{k-1}\left[1+A\left(q^{j}\right)\left(W_{q^{j+1}}-W_{q^{j}}\right)\right] .
$$

\section{Conflicts of Interest}

The author declares that he has no conflicts of interest.

\section{Acknowledgments}

The author would like to express his sincere gratitude to Professor Martin Bohner for inviting him to present this work at the Time Scales Seminar, Missouri S\&T, on October 5, 2016, and also for pointing out the use of delta (Hilger) derivatives. He would also like to thank Professor David Grow for an inspiring discussion on the topic. Special thanks are dedicated to Missouri University of Science and Technology (formerly University of Missouri, Rolla) for a startup fund that supports this work.

\section{References}

[1] M. Bohner and A. Peterson, Dynamical Equations on Time Scales, An Introduction with Applications, Birkhäuser Boston, Boston, Mass, USA, 2001.

[2] M. Bohner, O. M. Stanzhytskyi, and A. O. Bratochkina, "Stochastic dynamic equations on general time scales," Electronic Journal of Differential Equations, no. 57, pp. 1-15, 2013.

[3] M. Bohner and S. Sanyal, "The stochastic dynamic exponential and geometric Brownian motion on isolated time scales," Communications in Mathematical Analysis, vol. 8, no. 3, pp. 120$135,2010$.

[4] S. Sanyal and D. Grow, "Existence and uniqueness for stochastic dynamic equations," International Journal of Statistics and Probability, vol. 2, no. 2, 2013.

[5] D. Grow and S. Sanyal, "Brownian motion indexed by a time scale," Stochastic Analysis and Applications, vol. 29, no. 3, pp. 457-472, 2011.

[6] N. H. Du and N. T. Dieu, "The first attempt on the stochastic calculus on time scale," Stochastic Analysis and Applications, vol. 29, no. 6, pp. 1057-1080, 2011.

[7] D. Grow and S. Sanyal, "The quadratic variation of Brownian motion on a time scale," Statistics and Probability Letters, vol. 82, no. 9, pp. 1677-1680, 2012.

[8] S. Bhamidi, S. N. Evans, R. Peled, and P. Ralph, "Brownian motion on time scales, basic hypergrometric functions, and some continued fractions of Ramanujan," IMS Collections, vol. 2, pp. 42-75, 2008.

[9] T. Kumagai, "Short time asymptotic behaviour and large deviation for Brownian motion on some affine nested fractals," Publications of the Research Institute for Mathematical Sciences, vol. 33, no. 2, pp. 223-240, 1997.

[10] G. Ben Arous and T. Kumagai, "Large deviations for Brownian motion on the Sierpinski gasket," Stochastic Processes and Their Applications, vol. 85, no. 2, pp. 225-235, 2000.
[11] R. Cont and D.-A. Fournié, "Change of variable formulas for non-anticipative functionals on path space," Journal of Functional Analysis, vol. 259, no. 4, pp. 1043-1072, 2010.

[12] E. Haven, "Quantum calculus ( $q$-calculus) and option pricing: a brief introduction," in Quantum Interaction, vol. 5494 of Lecture Notes in Computer Science, pp. 308-314, Springer, Berlin, Germany, 2009.

[13] E. Haven, "Itô's lemma with quantum calculus ( $q$-calculus): some implications," Foundations of Physics, vol. 41, no. 3, pp. 529-537, 2011.

[14] W. Bryc, "On integration with respect to the q-Brownian motion," Statistics \& Probability Letters, vol. 94, pp. 257-266, 2014.

[15] R. P. Agarwal and M. Bohner, "Basic calculus on time scales and some of its applications," Results in Mathematics, vol. 35, no. 1-2, pp. 3-22, 1999. 


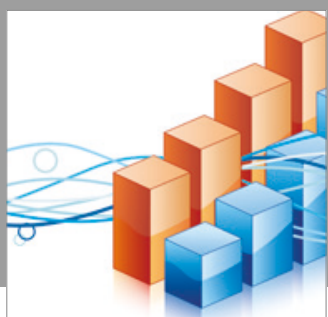

Advances in

Operations Research

vatersals

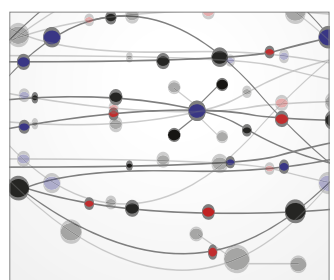

\section{The Scientific} World Journal
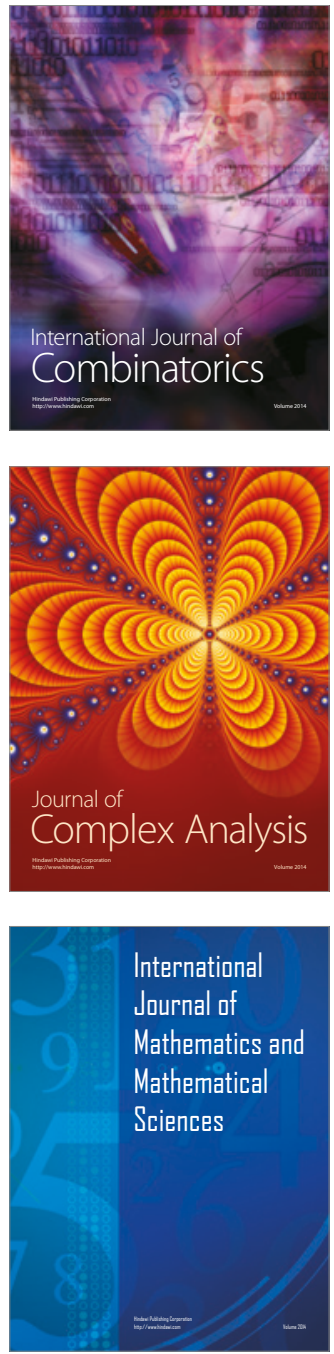
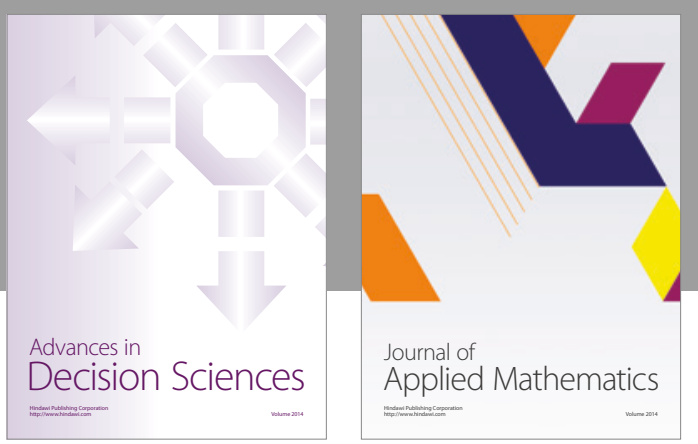

Algebra

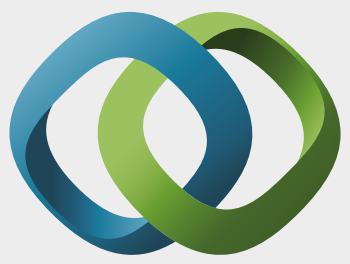

\section{Hindawi}

Submit your manuscripts at

https://www.hindawi.com
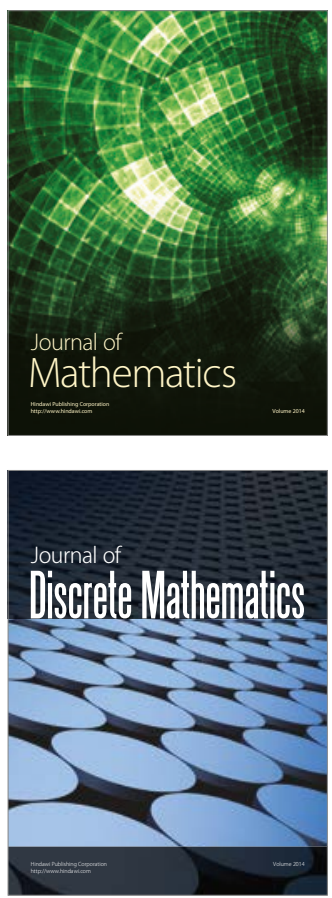

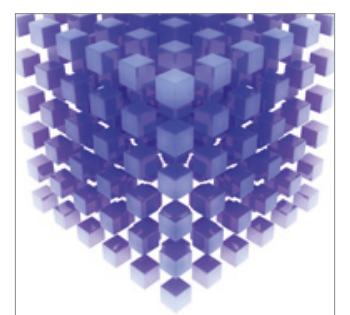

Mathematical Problems in Engineering
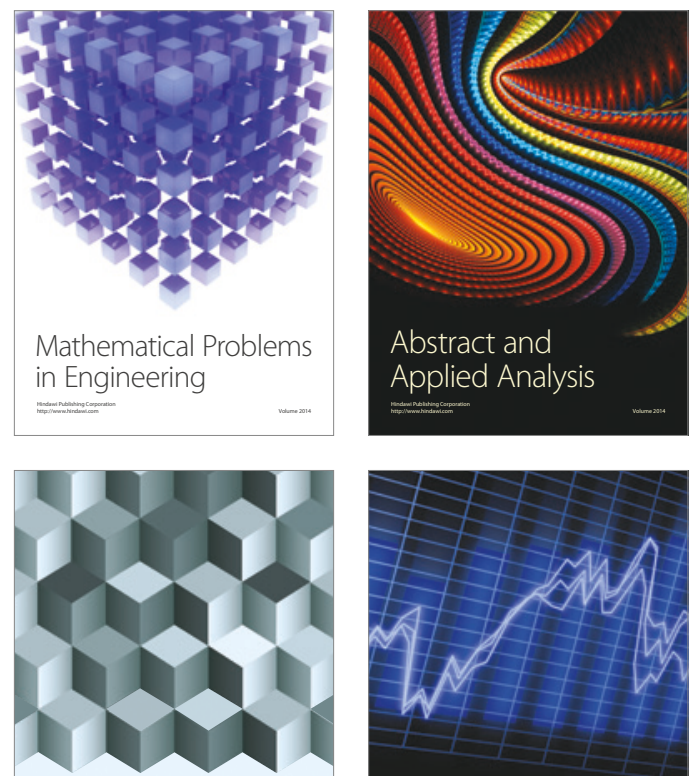

Journal of

Function Spaces

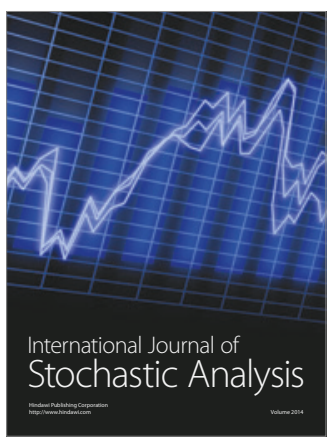

Probability and Statistics
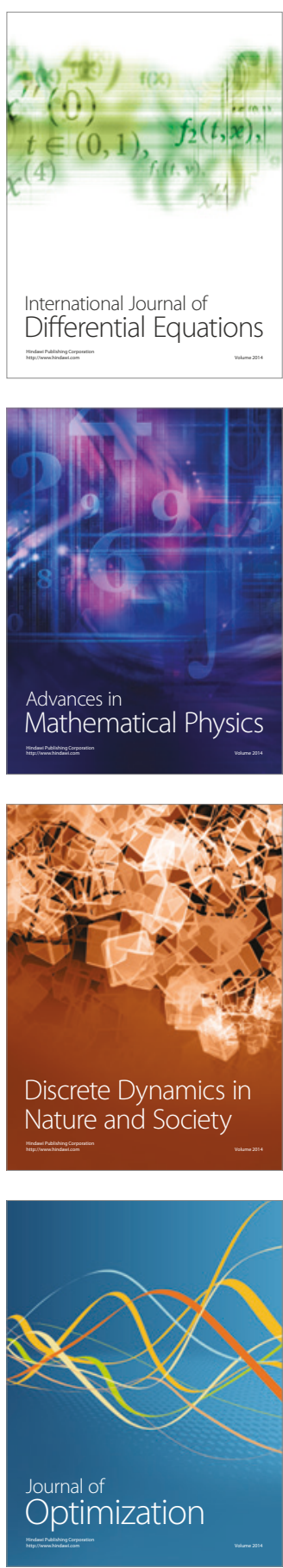